\title{
Article
}

\section{The Deepest Radio Observations of Nearby Type IA Supernovae: Constraining Progenitor Types and Optimizing Future Surveys}

Lundqvist, Peter, Kundu, Esha, Perez-Torres, Miguel, Ryder, Stuart D., Bjornsson, Claes-Ingvar, Moldon, Javier, Argo, Megan K., Beswick, Robert J., Alberdi, Antxon and Kool, Erik C.

Available at https://clok.uclan.ac.uk/31497/

Lundqvist, Peter, Kundu, Esha, Perez-Torres, Miguel, Ryder, Stuart D., Bjornsson, Claes-Ingvar, Moldon, Javier, Argo, Megan K. orcid iconORCID: 0000-0003-3594-0214, Beswick, Robert J., Alberdi, Antxon et al (2020) The Deepest Radio Observations of Nearby Type IA Supernovae: Constraining Progenitor Types and Optimizing Future Surveys. Astrophysical Journal, 890 (2). ISSN 0004-637X

It is advisable to refer to the publisher's version if you intend to cite from the work. http://dx.doi.org/10.3847/1538-4357/ab6dc6

For more information about UCLan's research in this area go to http://www.uclan.ac.uk/researchgroups/ and search for < name of research Group>.

For information about Research generally at UCLan please go to http://www.uclan.ac.uk/research/

All outputs in CLoK are protected by Intellectual Property Rights law, including Copyright law. Copyright, IPR and Moral Rights for the works on this site are retained by the individual authors and/or other copyright owners. Terms and conditions for use of this material are defined in the policies page. 


\title{
The Deepest Radio Observations of Nearby SNe Ia: Constraining Progenitor Types and Optimizing Future Surveys
}

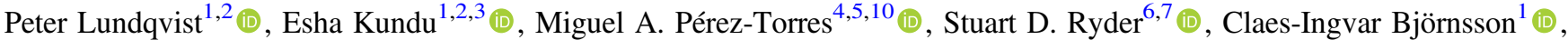 \\ Javier Moldon $^{4,8}$ (D), Megan K. Argo ${ }^{8,9}$ (D), Robert J. Beswick ${ }^{8}$, Antxon Alberdi ${ }^{4}$, and Erik C. Kool ${ }^{1,2,6,7}$ (D) \\ ${ }^{1}$ Department of Astronomy, AlbaNova University Center, Stockholm University, SE-10691 Stockholm, Sweden; peter@astro.su.se \\ ${ }^{2}$ The Oskar Klein Centre, AlbaNova, SE-10691 Stockholm, Sweden \\ ${ }^{3}$ International Centre for Radio Astronomy Research, Curtin University, Bentley, WA 6102, Australia \\ ${ }^{4}$ Instituto de Astrofísica de Andalucía, Glorieta de las Astronomía, s/n, E-18008 Granada, Spain \\ ${ }^{5}$ Departamento de Física Teorica, Facultad de Ciencias, Universidad de Zaragoza, Spain \\ ${ }^{6}$ Department of Physics and Astronomy, Macquarie University, Sydney NSW 2109, Australia \\ 7 Australian Astronomical Observatory, 105 Delhi Rd., North Ryde, NSW 2113, Australia \\ 8 Jodrell Bank Centre for Astrophysics, School of Physics and Astronomy, University of Manchester, M13 9PL, UK \\ ${ }^{9}$ Jeremiah Horrocks Institute, University of Central Lancashire, Preston PR1 2HE, UK \\ Received 2019 December 30; revised 2020 January 15; accepted 2020 January 16; published 2020 February 25
}

\begin{abstract}
We report deep radio observations of nearby Type Ia supernovae (SNe Ia) with the electronic Multi-Element Radio Linked Interferometer Network and the Australia Telescope Compact Array. No detections were made. With standard assumptions for the energy densities of relativistic electrons going into a power-law energy distribution and the magnetic field strength $\left(\epsilon_{\mathrm{e}}=\epsilon_{\mathrm{B}}=0.1\right)$, we arrive at upper limits on mass-loss rate for the progenitor system of SN 2013dy (SN 2016coj, SN 2018gv, SN 2018pv, SN 2019np) of $\dot{M} \lesssim 12(2.8,1.3,2.1,1.7) \times 10^{-8} M_{\odot} \mathrm{yr}^{-1}\left(v_{w} / 100 \mathrm{~km} \mathrm{~s}^{-1}\right)$, where $v_{w}$ is the wind speed of the mass loss. To SN 2016coj, SN 2018gv, SN 2018pv, and SN 2019np we add radio data for 17 other nearby SNe Ia and model their nondetections. With the same model as described, all 21 SNe Ia have $\dot{M} \lesssim 4 \times 10^{-8} M_{\odot} \mathrm{yr}^{-1}\left(v_{w} / 100 \mathrm{~km} \mathrm{~s}^{-1}\right)$. We compare those limits with the expected mass-loss rates in different single-degenerate progenitor scenarios. We also discuss how information on $\epsilon_{\mathrm{e}}$ and $\epsilon_{\mathrm{B}}$ can be obtained from late observations of SNe Ia and the youngest SN Ia remnant detected in radio, G1.9+0.3, as well as strippedenvelope core-collapse SNe. We highlight SN 2011dh and argue for $\epsilon_{\mathrm{e}} \approx 0.1$ and $\epsilon_{\mathrm{B}} \approx 0.0033$. Finally, we discuss strategies to observe at radio frequencies to maximize the chance of detection, given the time since explosion, the distance to the $\mathrm{SN}$, and the telescope sensitivity.
\end{abstract}

Unified Astronomy Thesaurus concepts: Supernova remnants (1667); Type Ia supernovae (1728); White dwarf stars (1799); Stellar mass loss (1613); Radio continuum emission (1340)

\section{Introduction}

Type Ia supernovae ( $\mathrm{SNe}$ Ia) have proven to be of fundamental importance as cosmological distance indicators (e.g., Riess et al. 1998; Perlmutter et al. 1999). Even so, we are still ignorant regarding what progenitor scenario is the correct one for the majority of SNe Ia. This compromises their use for precision cosmology. In addition, they are key players in the chemical evolution of galaxies, but not knowing the details of progenitor evolution, the explosion, and the nucleosynthesis means that we do not fully understand the timescale over which $\mathrm{SNe}$ Ia turn on, adding uncertainty to models for the chemical enrichment in the universe.

It is a generally accepted fact that $\mathrm{SNe}$ Ia are thermonuclear explosions of white dwarfs (WDs; Hoyle \& Fowler 1960). There are mainly two competing classes of models leading to an SN Ia thermonuclear explosion. One is the doubledegenerate (DD) model, where two WDs merge and explode (e.g., Tutukov \& Yungelson 1979; Iben \& Tutukov 1984; Webbink 1984; Thompson 2011; Maoz et al. 2014). The other is the single-degenerate (SD) model, where the companion is a nondegenerate star (e.g., Whelan \& Iben 1973; Nomoto 1982; Wang 2018). Here the WD accretes matter from the companion until it undergoes unstable runaway nuclear burning. A branch

\footnotetext{
${ }^{10}$ Visiting Scientist.
}

of these models is the so-called spun-up/spun-down superChandrasekhar mass WDs (Di Stefano et al. 2011; Justham 2011), where mass transfer is no longer active at the time of explosion.

One way to discriminate among different progenitor models of $\mathrm{SNe}$ Ia is to obtain information about the circumstellar medium of the exploding star. In scenarios with mass transfer from a nondegenerate companion, nonconservative mass transfer will give rise to a circumstellar medium (see, e.g., Branch et al. 1995) with a structure that depends on the massloss history of the system. When the SN ejecta are expelled into this medium, a shock is bound to form, resulting in radio and X-ray emission (Chevalier 1982b). In the DD scenario, the surrounding medium is likely to be of interstellar origin, and also in the SD spun-up/spun-down scenario one can expect a low-density medium in the vicinity of the progenitor. In these two scenarios, essentially no radio or X-ray emission is expected.

Several early attempts were made to detect radio (e.g., Panagia et al. 2006; Hancock et al. 2011) and X-ray (e.g., Hughes et al. 2007; Russell \& Immler 2012) emission from SNe Ia. These searches were hampered by their limited sensitivity and some inadequate assumptions for the modeling. The situation improved with the emergence of the very nearby SN 2011fe and SN 2014J, for which sensitive observations could be made. Using methods of interpretation incorporated 
from stripped-envelope $\mathrm{SNe}$, upper limits on the mass-loss rate from the progenitor systems have been obtained. Radio and X-ray limits for these two SNe Ia suggest $\dot{M} \lesssim 10^{-9} M_{\odot} \mathrm{yr}^{-1}$ (Chomiuk et al. 2012, 2016; Pérez-Torres et al. 2014) and $\dot{M} \lesssim 2 \times 10^{-9} M_{\odot} \mathrm{yr}^{-1}$ (Margutti et al. 2012, 2014), respectively, assuming a wind velocity of $100 \mathrm{~km} \mathrm{~s}^{-1}$. In addition to this, Chomiuk et al. (2016) have compiled a very comprehensive list of deep observations with the Jansky Very Large Array (JVLA) of nearby SNe Ia. Here we report five more $\mathrm{SNe}$ Ia to add to this list from our ongoing programs on the electronic Multi-Element Radio Linked Interferometer Network (eMERLIN) and the Australia Telescope Compact Array (ATCA), namely, SN 2013dy, SN 2016coj, SN 2018gv, SN 2018pv, and SN 2019np. Like in previous attempts, for other $\mathrm{SNe}$ Ia, we do not detect these five $\mathrm{SNe}$ in the radio.

The nondetections of radio and X-ray emission from $\mathrm{SNe}$ Ia have added to a growing consensus that SNe Ia mainly stem from DD explosions (e.g., Maoz et al. 2014), but a potential problem is that no obvious candidate system with double WDs has ever been identified (Rebassa-Mansergas et al. 2019). This, however, seems consistent with the intrinsic faintness of these objects. For potential SD progenitors, one should not disregard the SD spun-up/spun-down scenario and/or that the generation of radio and X-ray emission could be less efficient than hitherto assumed. Also, there is, in fact, evidence of circumstellar material from time-varying absorption features in the $\mathrm{NaI} D$ line for some SNe Ia (Patat et al. 2007; Simon et al. 2009). The exact location of this material is still debated, and there is no support for the idea that shells around $\mathrm{SNe}$ Ia, which give rise to dust scattering, are of circumstellar origin (Bulla et al. 2018).

There is a subset of SNe Ia that indeed show clear evidence of circumstellar interaction, the first ones being SN 2002ic (Hamuy et al. 2003) and SN 2005gj (Aldering et al. 2006), and the first case with both circumstellar interaction and timevarying narrow absorption lines was PTF $11 \mathrm{kx}$ (Dilday et al. 2012). The most recently reported circumstellar interaction examples are SN 2015cp (Graham et al. 2019) and SN 2018fhw (Valley et al. 2019). All these show Balmer line emission, so their progenitor systems are with little doubt of SD origin. Graham et al. (2019) estimate that $<6 \%$ of all SNe Ia have circumstellar shells within $3 \times 10^{17} \mathrm{~cm}$ from the exploding star. Due to selection effects, this fraction could be even smaller.

At some time after the explosion, the $\mathrm{SN}$ will turn on as a radio source, even if one has to wait until the supernova remnant (SNR) stage. A local example is G1.9+0.3, and there is also a hint that SN 1885A in Andromeda may now be visible at radio wavelengths (Sarbadhicary et al. 2019). We discuss the information we can gain from these to use in models for young $\mathrm{SNe}$ Ia.

Here we first describe the radio observations of SN 2013dy, SN 2016coj, SN 2018gv, SN 2018pv, and SN 2019np (Section 2), and in Section 3 we discuss the model we are using to interpret the observations. In Section 4 we summarize the results for the five SNe. Then, in Section 5, we choose the 21 best-observed SNe Ia in radio, along with the youngest local SN Ia remnant seen in radio (SNRG1.9+0.3), to draw some conclusions about what radio observations of $\mathrm{SNe}$ Ia can actually constrain in terms of the nature of the progenitor system. We also discuss optimal strategies for observing $\mathrm{SNe}$ Ia in terms of time since explosion, radio frequency, and sensitivity. Finally, we wrap up the paper in Section 6 with our main conclusions.

\section{Observations and Data Reduction}

The data for our observations of the five nearby SNe Ia SN 2013dy, SN 2016coj, SN 2018gv, SN 2018pv, and SN 2019np are collected in Tables 1 and 2. Here we describe these observations.

\subsection{SN 2013dy}

We observed SN 2013dy in the nearby $(D=13.7 \mathrm{Mpc})$ galaxy NGC 7250 with the electronic Multi-Element Radio Linked Interferometer Network (e-MERLIN; Pérez-Torres et al. 2013). SN 2013dy was discovered on 2013 July 10.45 UT (Casper et al. 2013; Zheng et al. 2013), and our radio observations were carried out during 2013 August 4-6, about 1 week after the $\mathrm{SN}$ had reached its $B$-band maximum. We observed SN 2013dy with e-MERLIN at a central frequency of $5.09 \mathrm{GHz}$ and used a total bandwidth of $512 \mathrm{MHz}$, which resulted in a synthesized Gaussian beam of 0 ." $13 \times 0$." 11 . We centered our observations at the position of the optical discovery and followed standard calibration and imaging procedures. We imaged a $20^{\prime \prime} \times 20^{\prime \prime}$ region centered at this position, after having stacked all our data. We found no evidence of radio emission above a $3 \sigma$ limit of $300 \mu \mathrm{Jy}_{\text {beam }}{ }^{-1}$ in a circular region of $1^{\prime \prime}$ in radius, centered at the $S N$ position. This value corresponds to an upper limit of the monochromatic $5.0 \mathrm{GHz}$ luminosity of $6.7 \times 10^{25} \mathrm{erg} \mathrm{s}^{-1} \mathrm{~Hz}^{-1}(3 \sigma)$.

\subsection{SN 2016coj}

We observed SN 2016coj in the nearby $(D=20.1 \mathrm{Mpc})$ galaxy NGC 4125 with e-MERLIN on 2016 May 28.18 UT (MJD 57,536.18; Pérez-Torres et al. 2017) . Our observations were carried out on 2016 June 3-4, 1 week after the SN discovery and about 1 week before reaching its $V$-band maximum (Zheng et al. 2016, 2017). e-MERLIN observed at a central frequency of $1.51 \mathrm{GHz}$ and used a total bandwidth of $512 \mathrm{MHz}$, which resulted in a synthesized Gaussian beam of 0 !" $13 \times 0$ "! 12 . We centered our observations at the position of the optical discovery and imaged a $16^{\prime \prime} \times 16^{\prime \prime}$ region centered at this position. We found no evidence of radio emission in the region of SN 2016coj down to a $3 \sigma$ limit of $126 \mu \mathrm{Jy}_{\text {beam }}{ }^{-1}$, which corresponds to an upper limit of the monochromatic $1.51 \mathrm{GHz}$ luminosity of $6.1 \times 10^{25} \mathrm{erg} \mathrm{s}^{-1} \mathrm{~Hz}^{-1}(3 \sigma)$.

In our analysis we also include data from AMI and the Jansky VLA (JVLA). In addition to what is reported in Mooley et al. (2016), further data are tabulated here. ${ }^{11}$ These data cover epochs from 2016 June 3.86 to 13.81 , estimated to correspond to 15-25 days after explosion (see Table 2).

\section{3. $S N 2018 g v$}

We used the ATCA at 5.5 and $9.0 \mathrm{GHz}$ with $2 \mathrm{GHz}$ bandwidths on 2018 January 18.6 UT to observe SN 2018gv (Ryder et al. 2018) situated in the galaxy NGC 2525. This SN was discovered on 2018 January 15.681 UT by Koichi Itagaki (TNS discovery report no. 16498) and identified as an SN Ia by Bufano et al. (2018) and Siebert et al. (2018). The observations and data reduction followed the same procedures as outlined

\footnotetext{
${ }^{11}$ https://4pisky.org/atel-sn2016coj-20160627/
} 
Table 1

Parameters of Observed SNe Ia

\begin{tabular}{|c|c|c|c|c|c|c|}
\hline Supernova & $\begin{array}{c}\text { Date of Optical Max } \\
\text { (UT) }\end{array}$ & $\begin{array}{l}\text { SN Position } \\
(\mathrm{J} 2000.0)\end{array}$ & Host Galaxy & Host Type & $\begin{array}{c}\text { Distance } \\
(\mathrm{Mpc})\end{array}$ & SN References \\
\hline SN 2013dy & 2013 Jul 27.71 & $22: 18: 17.60,+40: 34: 09.6$ & NGC 7250 & $\mathrm{Sdm}$ & 13.7 & (1) \\
\hline SN 2016coj & 2016 Jun 08.35 & $12: 08: 06.80,+65: 10: 38.2$ & NGC 4125 & E pec & 20.1 & (2) \\
\hline SN 2018gv & 2018 Feb 3 & $08: 05: 34.61,-11: 26: 16.3$ & NGC 2525 & $\mathrm{SB}(\mathrm{s}) \mathrm{c}$ & 16.8 & (3) \\
\hline SN 2018pv & 2018 Feb 16 & $11: 52: 55.70,+36: 59: 11.6$ & NGC 3941 & $\mathrm{SB}(\mathrm{s})$ & 13.1 & (4) \\
\hline SN 2019np & 2019 Jan 26 & $10: 29: 21.96,+29.30 .38 .4$ & NGC 3254 & $\mathrm{SA}(\mathrm{s}) \mathrm{bc}$ & 22 & (5) \\
\hline
\end{tabular}

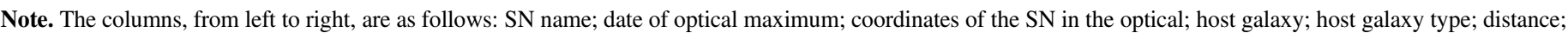

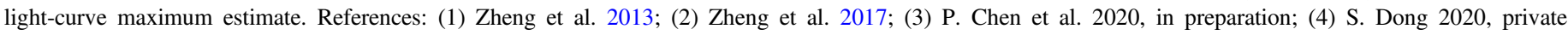
communication; (5) S. Dong and N. Elias-Rosa 2020, private communication.

for SN 2011hs by Bufano et al. (2014). No radio emission was detected down to $3 \sigma$ upper limits of $120 \mu \mathrm{Jy}$ beam $^{-1}$ at $5.5 \mathrm{GHz}$ and $30 \mu \mathrm{Jy}$ beam $^{-1}$ at $9.0 \mathrm{GHz}$. The total on-source time at each frequency was $6.8 \mathrm{hr}$. Adopting the host galaxy distance from Tully et al. (2013) of $16.8 \mathrm{Mpc}$, this implies an upper limit on the $9.0 \mathrm{GHz}$ luminosity of $1.0 \times 10^{25}$ $\mathrm{erg} \mathrm{s}^{-1} \mathrm{~Hz}^{-1}(3 \sigma)$, and four times higher at $5.5 \mathrm{GHz}$.

\subsection{SN 2018pv}

We observed the SN Ia SN 2018pv with e-MERLIN at $5.1 \mathrm{GHz}$ on 2018 February 3.63 UT (MJD 58,153.13) in the nearby $(z=0.0031)$ galaxy NGC 3941 (Tsuboi, TNS discovery report no. 16800). A spectrum on 2018 February 8.78 (MJD 58,158.78) confirmed the $\mathrm{SN}$ as a Type Ia event a few days before maximum (Yamanaka et al. 2018). Our observations (Pérez-Torres et al. 2018) were carried out on 2018 February 9-10 UT (MJD 58,159.08), 6 days after the SN discovery. We centered our observations at the position of the optical discovery (see Table 1). We found no evidence of radio emission in a circular region of $4 . \prime 0$ diameter surrounding SN 2018pv, down to a $3 \sigma$ upper limit of $57.6 \mu \mathrm{Jy}_{\text {beam }}{ }^{-1}$. For an assumed distance of $13.1 \mathrm{Mpc}$, the corresponding upper limit on the monochromatic $5.1 \mathrm{GHz}$ luminosity is $1.2 \times 10^{25}$ $\operatorname{erg~s}^{-1} \mathrm{~Hz}^{-1}(3 \sigma)$.

\subsection{SN 2019np}

We observed the SN Ia SN 2019np with e-MERLIN between 2019 January 14.81 and 15.46 UT (Pérez-Torres et al. 2019). SN 2019np was discovered on 2019 January 9.67 UT in the nearby $(z=0.00452)$ galaxy NGC 3254 (Itagaki, TNS discovery report no. 28550), and a spectrum on 2019 January 10.83 UT confirmed the SN as a Type Ia event 2 weeks before maximum (Burke, TNS classification report no. 3399). This is probably a lower limit since $B$-band maximum appears to have occurred around 2019 January 26 (S. Dong and N. Elias-Rosa 2020, private communication). Our observations were thus carried out 5 days after the SN discovery and $t \lesssim$ 10 days after the $\mathrm{SN}$ explosion. For a conservative estimate of $t$ we have used 10 days. We observed at a central frequency of $1.51 \mathrm{GHz}$, with a bandwidth of $512 \mathrm{MHz}$, and centered our observations at the position of the optical discovery (see Table 1). We found no evidence of radio emission in a circular region of 10 " 0 diameter surrounding SN 2019np, down to a $3 \sigma$ upper limit of $66 \mu \mathrm{Jy}_{\text {beam }^{-1}}$. For an assumed distance of $22 \mathrm{Mpc}$, the corresponding upper limit of the monochromatic $1.51 \mathrm{GHz}$ luminosity is $3.82 \times 10^{25} \mathrm{erg} \mathrm{s}^{-1} \mathrm{~Hz}^{-1}(3 \sigma)$. In our analysis we also include MeerKAT observations, commencing at 2019 January 11.97 UT (Heywood et al. 2019). The total integration lasted $3.25 \mathrm{hr}$ in the frequency band 856-1690 MHz. The observation resulted in a $3 \sigma$ upper limit of 57 $\mu \mathrm{Jy} \mathrm{beam}^{-1}$ at $1280 \mathrm{MHz}$, corresponding to $3.30 \times 10^{25}$ $\operatorname{erg~s}^{-1} \mathrm{~Hz}^{-1}(3 \sigma)$. We have used $t=7$ days, but this should be considered as an upper limit on $t$.

\section{Modeling the Radio Emission from SNe Ia}

We now interpret the upper limits on radio emission from the $\mathrm{SNe}$ in Section 2 within the framework of circumstellar interaction. The SN shock-wave expands out into its circumstellar gas, and a high-energy density shell forms. Here electrons are accelerated to relativistic speeds, and significant magnetic fields are generated. The relativistic electrons radiate synchrotron emission (e.g., Chevalier 1982b), which we probe with our radio observations.

We use the same model for the radio emission as in PérezTorres et al. (2014) and Kundu et al. (2017). In particular, we assume that electrons are accelerated to relativistic energies, with a power-law distribution, $d N / d E=N_{0} E^{-p}$, where $E=\gamma m_{e} c^{2}$ is the energy of the electrons and $\gamma$ is the Lorentz factor. For synchrotron emission, the intensity of optically thin emission is $\propto \nu^{-\alpha}$, where $\alpha=(p-1) / 2$. As shown for Type Ibc SNe, $\alpha \approx 1$ (Chevalier \& Fransson 2006), and we therefore use $p=3$ as our default value.

The density of the ambient medium as a function of radial distance, $r$, can be given as $\rho(r)=n_{\mathrm{CSM}}(r) \mu$, where $n_{\mathrm{CSM}}(r)$ and $\mu$ are the particle density and mean atomic weight of the surrounding gas, respectively. In the case of a constant-density medium we put $n_{\mathrm{CSM}}(r)=n_{0}$, and for a wind medium $\rho(r) \propto r^{-s}$. For constant $\dot{M} / v_{w}$, where $\dot{M}$ and $v_{w}$ are the mass-loss rate of the progenitor and the velocity at which this mass has been ejected from the system, respectively, $\rho(r)=\dot{M} /\left(4 \pi r^{2} v_{w}\right)$. In our models, we test the two scenarios $s=0$ and $s=2$.

For the SN ejecta, we resort to two models, also discussed in Kundu et al. (2017). One is called the N100 model (Röpke et al. 2012; Seitenzahl et al. 2013) and tests the SD scenario. This is a delayed detonation model where the central region is ignited by 100 sparks. The other is known as a violent merger model (Pakmor et al. 2012), which probes the DD channel. In this, two $\mathrm{C} / \mathrm{O}$ degenerate stars with masses of 1.1 and $0.9 M_{\odot}$ merge and produce a successful SN explosion. The total masses and asymptotic kinetic energies of the ejecta for $\mathrm{N} 100$ and the violent merger model are 1.4 and $1.95 M_{\odot}$ and $1.45 \times 10^{51}$ and $1.7 \times 10^{51} \mathrm{erg}$, respectively.

For both these models, the density profiles of the ejecta are given by the numerical simulations up to around a velocity of 
Table 2

Observations of Studied Supernovae

\begin{tabular}{|c|c|c|c|c|c|c|c|c|c|}
\hline SN Name & $\begin{array}{l}\text { Observation } \\
\text { Date } \\
\text { (UT) }\end{array}$ & Facility & $\begin{array}{l}\text { Central } \\
\text { Freq. } \\
(\mathrm{GHz})\end{array}$ & $\begin{array}{c}\text { Time since } \\
\text { Explosion } \\
\text { (Days) }\end{array}$ & $\begin{array}{c}\text { Flux } \\
\text { Density }(1 \sigma) \\
(\mu \mathrm{Jy})\end{array}$ & $\begin{array}{c}\text { Luminosity } \\
\text { Upper Limit }(3 \sigma) \\
\left(10^{25} \mathrm{erg} \mathrm{s}^{-1} \mathrm{~Hz}^{-1}\right)\end{array}$ & $\begin{array}{c}\dot{M} / v_{w} \\
\text { Upper Limit } \\
\left(\frac{10^{-8} M_{\odot} \mathrm{yr}^{-1}}{100 \mathrm{~km} \mathrm{~s}^{-1}}\right) \\
\end{array}$ & $\begin{array}{c}n_{0} \\
\text { Upper Limit } \\
\left(\mathrm{cm}^{-3}\right)\end{array}$ & References \\
\hline \multirow[t]{9}{*}{ SN 2016coj } & 2016 Jun 3.42 & e-MERLIN & 1.51 & 11 & 42 & 6.09 & 2.8 & 240 & (1), (3) \\
\hline & 2016 Jun 3.86 & AMI & 15.0 & 11 & 101 & 14.6 & 18 & 2300 & (4) \\
\hline & 2016 Jun 5.89 & AMI & 15.0 & 13 & 74 & 10.7 & 17 & 1600 & (4) \\
\hline & & JVLA & 4.5 & 18 & 20 & 2.90 & 4.5 & 180 & (4) \\
\hline & & JVLA & 7.4 & 18 & 16 & 2.32 & 5.4 & 220 & (4) \\
\hline & & JVLA & 8.5 & 18 & 17 & 2.42 & 6.1 & 260 & (4) \\
\hline & & JVLA & 10.9 & 18 & 18 & 2.56 & 7.4 & 330 & (4) \\
\hline & & JVLA & 13.5 & 18 & 13 & 1.93 & 7.1 & 310 & (4) \\
\hline & & JVLA & 16.5 & 18 & 16 & 2.36 & 9.3 & 420 & (4) \\
\hline SN 2018pv & 2018 Feb 9.23 & e-MERLIN & 5.1 & 14 & 19 & 1.18 & 2.1 & 120 & $(1),(6)$ \\
\hline \multirow[t]{2}{*}{ SN 2019np } & 2019 Jan 11.97 & MeerKAT & 1.28 & 7 & 19 & 3.30 & $(1.8)^{\mathrm{a}}$ & 220 & (7) \\
\hline & 2019 Jan 14.81 & e-MERLIN & 1.51 & 10 & 22 & 3.82 & 1.7 & 160 & (1), (8) \\
\hline
\end{tabular}

Notes. The columns, from left to right, are as follows: SN name; starting observing time, UT; observational facility; central frequency in GHz; mean observing epoch (in days since explosion); $1 \sigma$ flux density upper limits, in $\mu \mathrm{Jy}$; the corresponding $3 \sigma$ spectral luminosity in units of $10^{25} \mathrm{erg} \mathrm{s}^{-1} \mathrm{~Hz}^{-1}$; inferred $3 \sigma$ upper limit to the mass-loss rate in units of $10^{-8} M_{\odot} \mathrm{yr}^{-1}$, for an assumed wind velocity of $100 \mathrm{~km} \mathrm{~s}^{-1}$ (the values for $\dot{M}$ are for $\epsilon_{\mathrm{B}}=\epsilon_{\mathrm{e}}=0.1$ ); inferred $3 \sigma$ upper limit to the circumstellar density for a constant-density medium, in units of $\mathrm{cm}^{-3}$. References: (1) this paper; (2) Pérez-Torres et al. 2013; (3) Pérez-Torres et al. 2017; (4) Mooley et al. 2016, and data tabulated on the web as described in Section 2.2; (5) Ryder et al. 2018; (6) Pérez-Torres et al. 2018; (7) Heywood et al. 2019; (8) Pérez-Torres et al. 2019.

${ }^{\mathrm{a}}$ No solution exists (see Figure 4).

$2.5 \times 10^{4} \mathrm{~km} \mathrm{~s}^{-1}$. Therefore, for the extreme outer part of the exploded WD a power-law density structure is considered, i.e., $\rho_{e j} \propto r^{-n}$. In this study we have assumed $n=13$ (see Kundu et al. 2017, for a discussion on $n$ ).

The interaction of the supersonic SN ejecta with the almost stationary ambient medium creates two shock waves, known as forward and reverse shocks. In the shocked gas encapsulated by these shocks, relativistic particles are accelerated in the presence of magnetic fields, and synchrotron radiation is emitted at radio wavelengths. We assume that the radio emission comes from a spherical homogeneous shell and that the evolution of this shell is described by a self-similar solution (Chevalier 1982a).

For a polytropic gas with $\gamma=5 / 3$, the compression of the gas across the strong shock is $\eta=4$, and the post-shock thermal energy density is $u_{\mathrm{th}}=9 / 8 \rho(r) v_{s}^{2}(r)$, where $v_{s}(r)$ is the velocity of the forward shock at a given distance $r$. We assume that fractions of the thermal energy, $\epsilon_{\mathrm{e}}=u_{\mathrm{e}} / u_{\mathrm{th}}$ and $\epsilon_{\mathrm{B}}=u_{\mathrm{B}} / u_{\mathrm{th}}$, go into the energy densities of electrons $\left(u_{\mathrm{e}}\right)$ and magnetic fields $\left(u_{\mathrm{B}}=B^{2} /(8 \pi)\right)$, respectively. Here $B$ is the magnetic field strength. We assume that in the post-shock region all electrons get accelerated. However, with time only a fraction of the electrons, represented by $\epsilon_{\text {rel }}$, remains relativistic with energy $E>m_{e} c^{2}$, where $m_{e}$ and $c$ are the mass of electrons and velocity of light, respectively. These relativistic electrons are the ones that give rise to radio emission.

Following Pérez-Torres et al. (2014), we have in our models considered synchrotron self-absorption (SSA) as the sole absorption mechanism of this radiation (see also the discussion in Kundu et al. 2017). In the optically thin regime, from a shell of radius $r_{s}$ and thickness $\Delta r$, the luminosity can be written as follows:

$$
L_{\nu, \text { thin }}=\frac{8 \pi^{2} k T_{\text {bright }} \vartheta_{\nu} r_{s}^{2}}{c^{2} f\left(\frac{\nu_{\text {peak }}}{\nu_{\text {abs }}}\right)} \nu_{\text {abs }, 0}^{(p+3) / 2} \nu^{-(p-1) / 2},
$$

with

$$
\nu_{\mathrm{abs}, 0}=\left(2 \Delta r \varkappa(p) N_{0} B^{(p+2) / 2}\right)^{2 /(p+4)}
$$

and

$$
f(x)=x^{1 / 2}\left[1-\exp \left(-x^{-(p+4) / 2}\right)\right]
$$

(Pérez-Torres et al. 2014; Kundu et al. 2017), where $k$ and $T_{\text {bright }}$ represent the Boltzmann constant and the brightness temperature, respectively. In this work it is assumed that $T_{\text {bright }}=5 \times 10^{10} \mathrm{~K}$, which is the same value as that considered in Pérez-Torres et al. (2014) and Kundu et al. (2017). Note that $T_{\text {bright }}$ is defined from the intensity at $\nu_{\mathrm{abs}, 0}$ (see Björnsson \& Lundqvist 2014). $\vartheta_{\nu}=\frac{L_{\nu}}{4 \pi^{2} r_{s}^{2} I_{\nu}(0)}$, with $I_{\nu}(0)$ being the intensity of radiation received from the equatorial plane of the SN, i.e., from that part of the shell for which path length is equal to $\Delta r$ along the line of sight. $\varkappa(p)$ and $B$ are the SSA coefficient and magnetic field strength in the post-shock region, respectively. For $n=13$ and $p=3$, the optically thin luminosity can be written for a constant-density medium, $s=0$, as

$$
L_{\nu \text {,hin }} \propto T_{\text {bright }} \epsilon_{\mathrm{e}}^{1.71} \epsilon_{\mathrm{B}}^{1.07} n_{0}^{1.28} t^{0.91},
$$


and for a wind medium with $s=2$ as

$$
L_{\nu, \text { thin }} \propto T_{\text {bright }} \epsilon_{\mathrm{e}}^{1.71} \epsilon_{\mathrm{B}}^{1.07}\left(\dot{M} / v_{w}\right)^{1.51} t^{-1.42} .
$$

\section{Results}

\subsection{Modeling the Data for Our Sample}

Radio emission from $\mathrm{SNe}$ Ia is attenuated by free-free absorption (FFA) in the external unshocked circumstellar medium and by SSA. In early analyses of SNe Ia (e.g., Panagia et al. 2006; Hancock et al. 2011), FFA was considered to dominate the absorption. However, more recent papers (Chomiuk et al. 2012, 2016; Horesh et al. 2012; Pérez-Torres et al. 2014; Kundu et al. 2017) conclude that FFA is insignificant. As discussed in Pérez-Torres et al. (2014), the free-free optical depth, $\tau_{\text {ff }}$, for a fully ionized wind at $10^{4} \mathrm{~K}$ and moving at $v_{w}=100 \mathrm{~km} \mathrm{~s}^{-1}$ is $\tau_{\mathrm{ff}} \sim 10^{-4} \lambda^{2}\left(\dot{M} / 10^{-7} M_{\odot} \mathrm{yr}^{-1}\right)^{2}\left(r_{s} / 10^{15} \mathrm{~cm}\right)^{-3}$, where $\lambda$ is in $\mathrm{cm}$. From our calculations, using the N100 model, the shock radius is at $\sim 10^{15} \mathrm{~cm}$ already at $\sim 2$ days for $\dot{M}=10^{-7}\left(v_{w} / 100 \mathrm{~km} \mathrm{~s}^{-1}\right) M_{\odot} \mathrm{yr}^{-1}$, which means that $\tau_{\text {ff }} \sim 3 \times 10^{-3}\left(\dot{M} / 10^{-7} M_{\odot} \mathrm{yr}^{-1}\right)^{2}$ at $5.5 \mathrm{GHz}$ at that epoch. Considering that X-ray nondetections for SN 2011fe and SN 2014J (Margutti et al. 2012, 2014) have put limits on $\dot{M} / v_{w}$ of order $10^{-9} M_{\odot} \mathrm{yr}^{-1}$ for $v_{w}=100 \mathrm{~km} \mathrm{~s}^{-1}$ for these SNe Ia, it is not a bold assumption that FFA can be neglected for normal SNe Ia. Horesh et al. (2012) used a similar argument to dismiss FFA in their analysis of radio emission from SN $2011 \mathrm{fe}$. In what follows, we only discuss frequencies higher than $1 \mathrm{GHz}$ and concentrate on $\dot{M} \lesssim 10^{-7}\left(v_{w} / 100 \mathrm{~km} \mathrm{~s}^{-1}\right) M_{\odot} \mathrm{yr}^{-1}$ and $t$ $\gtrsim 2$ days, and therefore only consider SSA.

We have used the model in Section 3 to calculate the expected emission from a circumstellar medium created by a wind (the $s=2$ case) and for a constant-density medium (the $s=0$ case). Expressions for epochs when SSA is negligible are given by Equations (4) and (5). These expressions can be used to study the dependence between the various parameters and are in most cases sufficient in order to estimate $\dot{M} / v_{w}$ and $n_{0}$. However, SSA can be important at very early epochs and especially at low frequencies, so the expressions for optically thin synchrotron emission may underestimate $\dot{M} / v_{w}$ and $n_{0}$. As discussed in Section 3, our models do include SSA.

\subsubsection{The Constant-density Case, $\mathrm{s}=0$}

We have used the merger model and methods discussed in Section 3 to estimate $n_{0}$ for SN 2013dy, SN 2016coj, SN $2018 \mathrm{gv}$, SN 2018pv, and SN 2019np. As shown in Table 2, the lowest limit on $n_{0}$ for those SNe Ia is $n_{0} \gtrsim 120 \mathrm{~cm}^{-3}$ (for SN 2016coj and SN 2018pv), assuming $\epsilon_{\mathrm{B}}=\epsilon_{\mathrm{e}}=0.1$. This is significantly higher than the density expected in the DD scenario, which is that of the interstellar medium, i.e., $\lesssim 1 \mathrm{~cm}^{-3}$. This shows that early radio observations of SNe Ia do not provide stringent limits on $n_{0}$, unless they are significantly closer than $20 \mathrm{Mpc}$.

As the radio luminosity in the $s=0$ case is expected to increase with time (e.g., Chomiuk et al. 2012; Pérez-Torres et al. 2014; Kundu et al. 2017), radio observations at late epochs constrain $n_{0}$ better (see, e.g., Chomiuk et al. 2016). For events nearby enough, like SN 2011fe and SN 2014J, tight limits on both $n_{0}$ and the microphysics parameters $\epsilon_{\mathrm{B}}$ and $\epsilon_{\mathrm{e}}$ can be obtained (Kundu et al. 2017; see also Section 5.3). Modeling data from the epochs of 1468 and 410 days and assuming

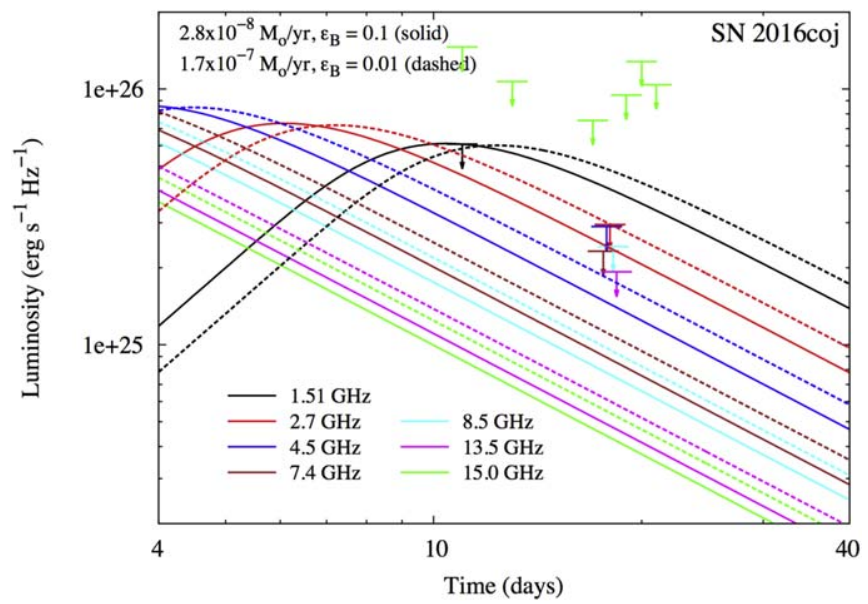

Figure 1. Radio data for SN 2016coj (see Table 2), together with models at various frequencies for an $s=2$ wind. Models use $\dot{M}=2.8(17) \times 10^{-8} M_{\odot} \mathrm{yr}^{-1}\left(v_{w} / 100 \mathrm{~km} \mathrm{~s}^{-1}\right)$ for $\epsilon_{\mathrm{B}}=0.1(0.01)$, with solid lines being for $\epsilon_{\mathrm{B}}=0.1$. Common parameters in both models are $\epsilon_{\mathrm{e}}=0.1, T_{\text {bright }}=5 \times 10^{10} \mathrm{~K}, n=13$, and $v_{w}=100 \mathrm{~km} \mathrm{~s}^{-1}$. Constraining observations are those at $1.51 \mathrm{GHz}$ on day 11 and at $2.7 \mathrm{GHz}$ on day 18 . Observations at different frequencies on day 18 have been shifted in steps of 0.2 days between 17.6 and 18.4 days to disentangle the data.

$\epsilon_{\mathrm{B}}=\epsilon_{\mathrm{e}}=0.1$, Kundu et al. (2017) find $n_{0} \lesssim 0.3 \mathrm{~cm}^{-3}$ for both SN 2011fe and SN 2014J, respectively. According to Chomiuk et al. (2016), limits for other SNe Ia do not come close to these numbers, the best cases being SN 1985A and SN 2012cg. Chomiuk et al. (2016) report $n_{0} \lesssim 13(10) \mathrm{cm}^{-3}$ for SN $1985 \mathrm{~A}$ (SN 2012cg) observed at $1.4(5.9) \mathrm{GHz}$ observations at 315 (216) days. For the sake of completeness, we have recalculated the corresponding values using our models and methods in Section 3, together with the data in Chomiuk et al. (2016) and using $\epsilon_{\mathrm{B}}=\epsilon_{\mathrm{e}}=0.1$. We find $n_{0} \lesssim 12(8) \mathrm{cm}^{-3}$ for SN 1985A (SN 2012cg), which is close to the numbers of Chomiuk et al. (2016).

\subsubsection{The Wind Case, $\mathrm{s}=2$}

While limits on $n_{0}$ in the $s=0$ for young SNe Ia case are of limited value, except for SN $2011 \mathrm{fe}$ and SN 2014J, early radio observations can be used to constrain $\dot{M} / v_{w}$ in the $s=2$ case with some stringency. As shown in Table 2, deep limits on $\dot{M} / v_{w}$ are obtained for SN 2016coj, SN 2018gv, SN 2018pv, and SN 2019np. For $\epsilon_{\mathrm{B}}=\epsilon_{\mathrm{e}}=0.1$, and using the N100 explosion model with $n=13$, we find upper limits of $\dot{M} \lesssim 2.8(1.3,2.1,1.7) \times 10^{-8} M_{\odot} \mathrm{yr}^{-1}\left(v_{w} / 100 \mathrm{~km} \mathrm{~s}^{-1}\right)$ for these four $\mathrm{SNe}$, respectively. The limit on $\dot{M} / v_{w}$ for SN 2013dy is about an order of magnitude larger.

We show modeled light curves for SN 2016coj in Figure 1, for SN 2018gv and SN 2018pv in Figure 2, and for SN 2019np in Figure 3. All models use $\epsilon_{\mathrm{e}}=0.1, T_{\text {bright }}=5 \times 10^{10} \mathrm{~K}$, and $n=13$, and we show results for both $\epsilon_{\mathrm{B}}=0.01$ and $\epsilon_{\mathrm{B}}=0.1$. For SN 2016coj, the most constraining data are from the e-MERLIN $1.51 \mathrm{GHz}$ observations on day 11 (see Table 2), but the JVLA data at $2.7 \mathrm{GHz}$ also provide stringent constraints. In particular, for $\epsilon_{\mathrm{B}}=0.01, \mathrm{SSA}$ is important at $1.51 \mathrm{GHz}$, while the optically thin $2.7 \mathrm{GHz}$ emission not only serves as an independent check but also sets a more stringent limit on $\dot{M} / v_{w}$. The mass-loss rate limit for the $\epsilon_{\mathrm{B}}=0.01$ case is $\dot{M} \lesssim 1.7 \times 10^{-7} M_{\odot} \mathrm{yr}^{-1}\left(v_{w} / 100 \mathrm{~km} \mathrm{~s}^{-1}\right)$, i.e., almost an order of magnitude higher than for $\epsilon_{\mathrm{B}}=0.1$. 


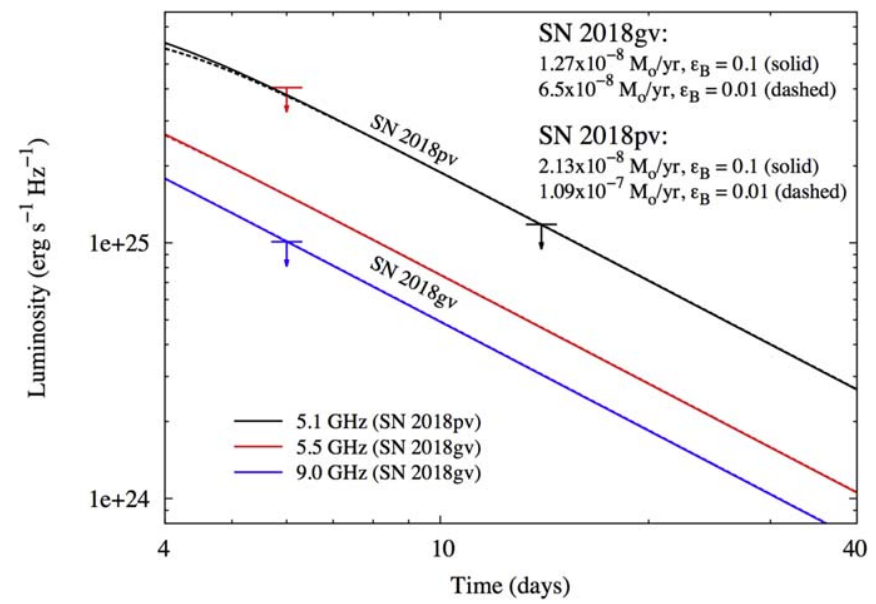

Figure 2. Radio data for SN 2018gv and SN 2018pv (see Table 2), together with models at various frequencies for an $s=2$ wind. Common model parameters are $\epsilon_{\mathrm{e}}=0.1, T_{\text {bright }}=5 \times 10^{10} \mathrm{~K}, n=13$, and $v_{w}=100 \mathrm{~km} \mathrm{~s}^{-1}$. Solid lines are for $\epsilon_{\mathrm{B}}=0.1$, and dashed lines are for $\epsilon_{\mathrm{B}}=0.01$. Note that dashed and solid lines overlap for SN 2018pv. The values for $\dot{M}$ in the different models are described in the figure. The constraining observations are at $5.1 \mathrm{GHz}$ for SN $2018 \mathrm{pv}$ and $9.0 \mathrm{GHz}$ for SN $2018 \mathrm{gv}$.

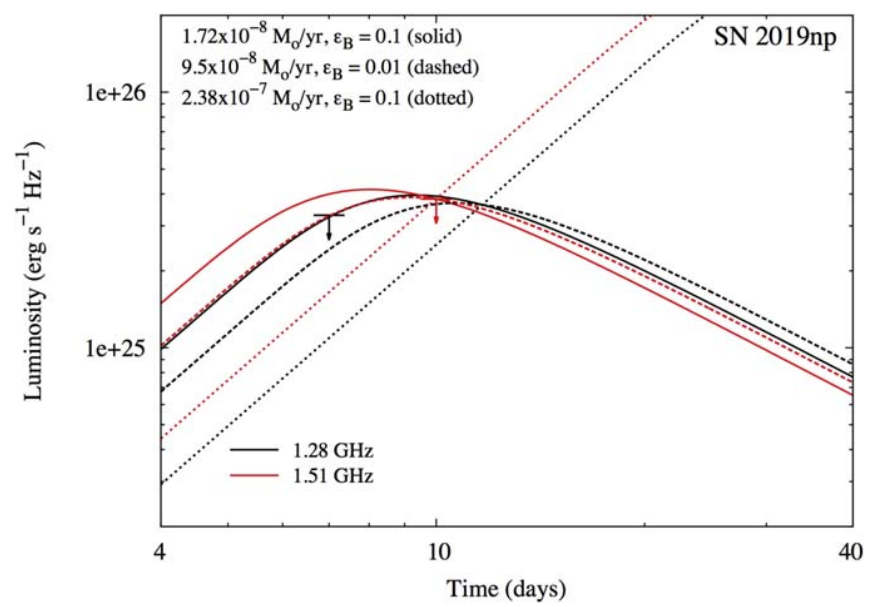

Figure 3. Radio data for SN 2019np (see Table 2), together with models at two frequencies for an $s=2$ wind. Common model parameters are $\epsilon_{\mathrm{e}}=0.1$, $T_{\text {bright }}=5 \times 10^{10} \mathrm{~K}, n=13$, and $v_{w}=100 \mathrm{~km} \mathrm{~s}^{-1}$. Solid and dotted lines are for $\epsilon_{\mathrm{B}}=0.1$, and dashed lines are for $\epsilon_{\mathrm{B}}=0.01$. The values for $\dot{M}$ in the different models are described in the figure. For $\epsilon_{\mathrm{B}}=0.1$ and $v_{w}=100 \mathrm{~km} \mathrm{~s}^{-1}$ $1.7 \times 10^{-8} M_{\odot} \mathrm{yr}^{-1} \leqslant \dot{M} \leqslant 2.4 \times 10^{-7} M_{\odot} \mathrm{yr}^{-1}$ is ruled out from the observation at $1.51 \mathrm{GHz}$.

In the models for SN 2018gv and SN 2018pv, SSA does not play a role for the 5-9 GHz light curves in Figure 2, not even for the models with $\epsilon_{\mathrm{B}}=0.01$. Models with $\epsilon_{\mathrm{B}}=0.1$ and $\epsilon_{\mathrm{B}}=0.01$ line up on top of each other, just by changing $\dot{M} / v_{w}$ by a factor of $10^{0.71} \approx 5.1$, as expected from Equation (5) for optically thin synchrotron radiation. The corresponding factor is larger $(\approx 6.7)$ for the marginally optically thick situation at $1.51 \mathrm{GHz}$ in Figure 1. The limits on $\dot{M} /\left(v_{w} / 100 \mathrm{~km} \mathrm{~s}^{-1}\right)$ for $\epsilon_{\mathrm{B}}=0.01$ and $\epsilon_{\mathrm{B}}=0.1$ for $\mathrm{SN} 2018 \mathrm{gv}$ and $\mathrm{SN} 2018 \mathrm{pv}$ are shown in Figure 2.

For SN 2019np, SSA is important at the low frequencies $(1.28$ and $1.51 \mathrm{GHz}$ ) used for observations of this $\mathrm{SN}$ (see Figure 3). For $1.28 \mathrm{GHz}$ at $t=7$ days, the peak luminosity for $\epsilon_{\mathrm{B}}=0.1$ is $3.25 \times 10^{25} \mathrm{erg} \mathrm{s}^{-1} \mathrm{~Hz}^{-1}$ and occurs for $\dot{M} \approx 1.8 \times 10^{-8}\left(v_{w} / 100 \mathrm{~km} \mathrm{~s}^{-1}\right) M_{\odot} \mathrm{yr}^{-1}$. This $1.28 \mathrm{GHz}$ luminosity is lower than the $3 \sigma$ limit listed in Table 2. To highlight this, we have put the upper limit on $\dot{M} / v_{w}$ for $1.28 \mathrm{GHz}$ in Table 2 in parentheses. For $1.51 \mathrm{GHz}$, at $t=10$ days, the modeled luminosity for $\epsilon_{\mathrm{B}}=0.1$ is higher than the observed $3 \sigma$ limit for $1.7 \times 10^{-8} M_{\odot} \mathrm{yr}^{-1} \lesssim \dot{M}\left(v_{w} / 100 \mathrm{~km} \mathrm{~s}^{-1}\right)^{-1} \lesssim-$

$2.4 \times 10^{-7} M_{\odot} \mathrm{yr}^{-1}$. The corresponding limits for $\epsilon_{\mathrm{B}}=0.01$ are

$9.5 \times 10^{-8} M_{\odot} \mathrm{yr}^{-1} \lesssim \dot{M}\left(v_{w} / 100 \mathrm{~km} \mathrm{~s}^{-1}\right)^{-1} \lesssim-$

$5.1 \times 10^{-7} M_{\odot} \mathrm{yr}^{-1}$.

For

$\dot{M}\left(v_{w} / 100 \mathrm{~km} \mathrm{~s}^{-1}\right)^{-1} \gtrsim 2.4(5.1) \times 10^{-7} M_{\odot} \mathrm{yr}^{-1} \quad$ and $\epsilon_{\mathrm{B}}=0.1(0.01) \mathrm{SSA}$ mutes the modeled $1.51 \mathrm{GHz}$ luminosity so that it becomes lower than the observed $3 \sigma$ luminosity limit. In Table 2 and in the following we have, however, treated $1.7 \times 10^{-8} M_{\odot} \mathrm{yr}^{-1}\left(v_{w} / 100 \mathrm{~km} \mathrm{~s}^{-1}\right)$ as a true upper limit for $\epsilon_{\mathrm{B}}=0.1$.

Figure 4 illustrates the relevance of SSA in probing $\dot{M} / v_{w}$ from SN Ia observations. We show, for a putative SN Ia at a distance of $D=15 \mathrm{Mpc}$, which minimum value of $\dot{M} / v_{w}$ can be probed, given the observing frequency, the time since explosion, and the flux limit. We have rescaled the flux density levels for the $\mathrm{SNe}$ marked in the figure to correspond to $D=15 \mathrm{Mpc}$. Solution curves for a given flux density level and vertical tick marks marking the time since explosion overlap for the $\dot{M} / v_{w}$ values tabulated in Tables 2 and 3. SSA attenuates the flux densities so that there is a minimum time since explosion when the $\mathrm{SN}$ can be detected for a given flux limit and observing frequency. For earlier times, SSA is so large that observations cannot constrain $\dot{M} / v_{w}$. In particular, there is no solution corresponding to the flux limit of the $1.28 \mathrm{GHz}$ observations at $t=7$ days for SN 2019np. This is also highlighted in Table 2, where $\dot{M} / v_{w}$ for the closest distance between the solution curve and the vertical line marking time since explosion in the panel has been put in parentheses. The situation is different for $1.51 \mathrm{GHz}$ at 10 days (middle panel; see also Table 3). Figure 4 provides a useful tool for selecting radio telescope facility and observing frequency for a newly detected SN Ia. For very young $\mathrm{SNe}$ (i.e., a few days old), the very lowest frequencies $(\lesssim 2 \mathrm{GHz})$ should be avoided, unless one can expect a $3 \sigma$ flux limit that is $\lesssim 10(D / 15 \mathrm{Mpc})^{-2} \mu \mathrm{Jy}$. For a 5 -day old SN Ia, the corresponding flux limit is $\lesssim 30(D / 15 \mathrm{Mpc})^{-2} \mu \mathrm{Jy}$.

\section{Discussion}

\subsection{Comparison to Previous Studies}

As discussed in Section 4.1.1, early radio data are often not useful to probe the $s=0$ scenario, and in the following we will mainly concentrate on the $s=2$ scenario. To put things in perspective, we have in Table 3 compiled all SNe Ia with the most constraining radio data for that scenario. Our four best cases, SN 2016coj, SN 2018gv, SN 2018pv, and SN 2019np, are the four most recent in this sample of $21 \mathrm{SNe}$ Ia. To form this sample, we have added to our SNe the ones with the lowest limits on $\dot{M} / v_{w}$ in the compilation of Chomiuk et al. (2016). In Table 3 we list upper limits on $\dot{M} / v_{w}$ using the same model as in Section 4.1.2 with $\epsilon_{\mathrm{B}}=0.1$. According to such an estimate, no $\mathrm{SN}$ in the sample has $\dot{M} \gtrsim 4.0 \times 10^{-8}\left(v_{w} / 100 \mathrm{~km} \mathrm{~s}^{-1}\right) M_{\odot} \mathrm{yr}^{-1}$. Seven SNe have $\dot{M} \lesssim 1.0 \times 10^{-8}\left(v_{w} / 100 \mathrm{~km} \mathrm{~s}^{-1}\right) M_{\odot} \mathrm{yr}^{-1}$, and they are SN 2011by, SN 2011ek, SN 2011fe, SN 2012cg, SN 2012fr, SN $2012 \mathrm{hr}$, and SN 2014J. SN 2011fe and SN 2014J have limits as low as $\dot{M} \lesssim 9 \times 10^{-10}\left(v_{w} / 100 \mathrm{~km} \mathrm{~s}^{-1}\right) M_{\odot} \mathrm{yr}^{-1}$. 

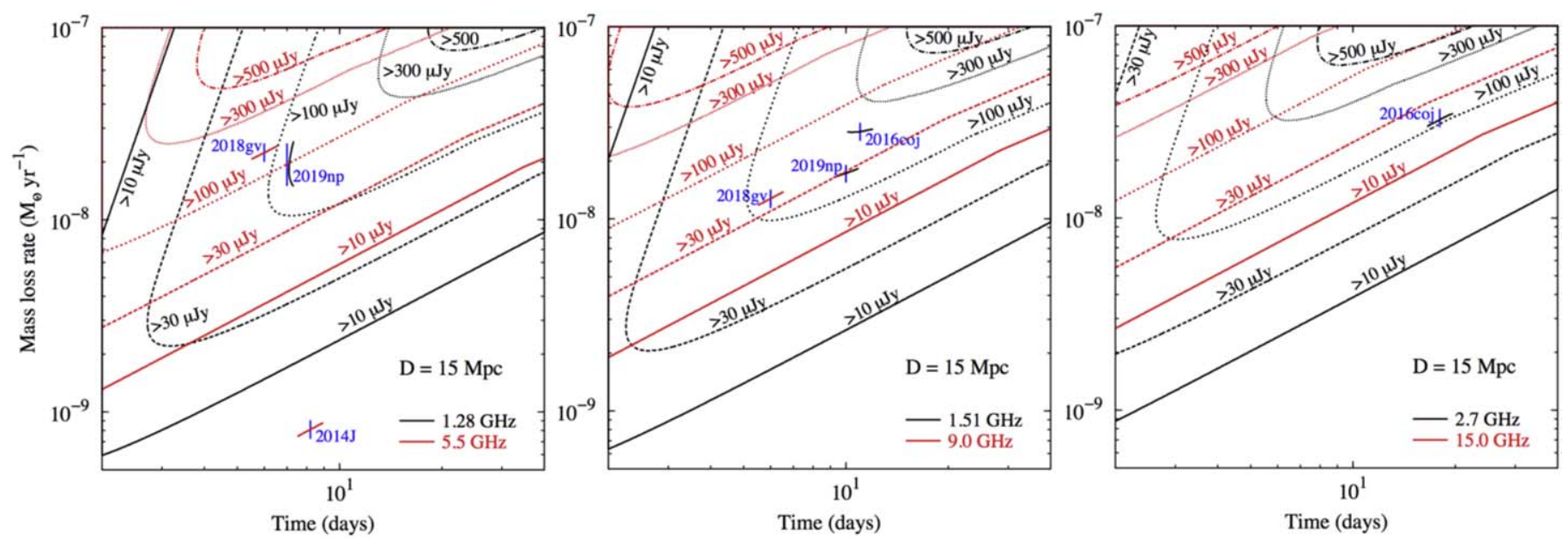

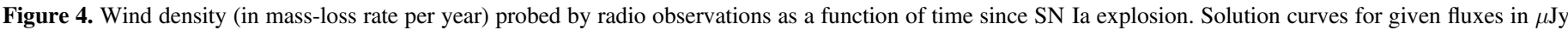

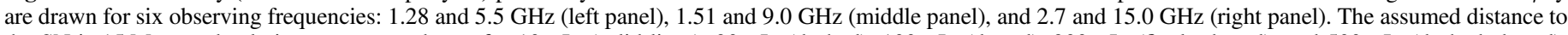

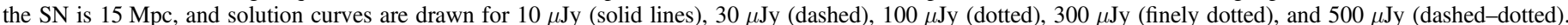

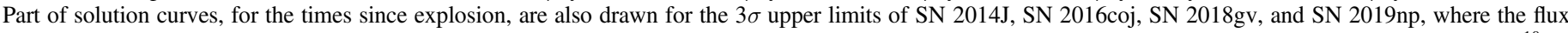

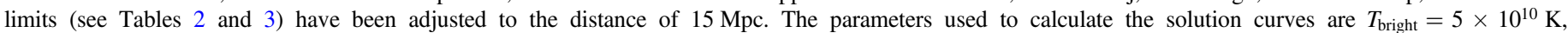

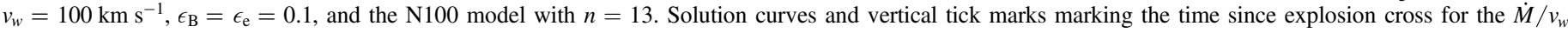

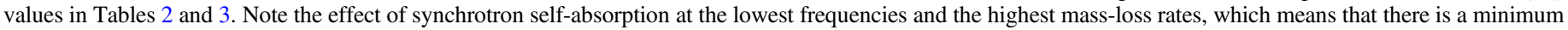
time since explosion when the SN can be detected for a given flux limit and observing frequency.

The limits on $\dot{M} / v_{w}$ in Table 3 (and used throughout this paper) were derived using the same distances to the $\mathrm{SNe}$ as in Section 2 and Chomiuk et al. (2016).

\subsection{Possible SD Progenitor Systems}

There are several possible SD scenarios, and all (except the so-called spun-up/spun-down super-Chandrasekhar mass scenario; see below) are characterized by a mass-loss rate and wind speed of the circumstellar gas expelled from the progenitor system. The expected mass-loss rate from the progenitor system, in decreasing order, includes symbiotic systems, WDs with steady nuclear burning, and recurrent novae. We have marked areas in Figure 5 (showing $\dot{M}$ vs. $v_{w}$ ) where possible SD progenitor systems reside. We have also marked (dashed lines) $3 \sigma$ limits on $\dot{M} / v_{w}$ from Table 3 for seven of the tabulated SNe, assuming $\epsilon_{\mathrm{B}}=\epsilon_{\mathrm{e}}=0.1, n=13$, $s=2, T_{\text {bright }}=5 \times 10^{10} \mathrm{~K}$, and the $\mathrm{N} 100$ explosion model. Areas in Figure 5 for the possible SD progenitor systems, lying below and to the right of the $3 \sigma$ limit dashed lines, are ruled out.

In symbiotic systems (red region in Figure 5), the WD accretes mass from a giant star (Hachisu et al. 1999), but the WD loses some of this matter at rates of $\dot{M} \gtrsim 10^{-8} M_{\odot} \mathrm{yr}^{-1}$ and velocity $v_{w} \approx 30 \mathrm{~km} \mathrm{~s}^{-1}$. From Figure 5 it is clear that this scenario is ruled out for all $\mathrm{SNe}$ in Table 3 with $\dot{M} \leqslant 1.7 \times 10^{-8} M_{\odot} \mathrm{yr}^{-1}\left(v_{w} /\left(100 \mathrm{~km} \mathrm{~s}^{-1}\right)\right)$, including our observed cases SN 2018gv and SN 2019np. This conclusion, however, rests on $\epsilon_{\mathrm{B}}=\epsilon_{\mathrm{e}}=0.1$, which is uncertain (see Section 5.3).

Circumstellar medium can also be created during Roche lobe overflow from a main-sequence, subgiant, helium, or giant star onto the WD. The expected rate is $3.1 \times 10^{-7} M_{\odot} \mathrm{yr}^{-1} \lesssim \dot{M}_{\mathrm{acc}} \lesssim 6.7 \times 10^{-7} M_{\odot} \mathrm{yr}^{-1} \quad$ (Nomoto et al. 2007). At those accretion rates, the WD experiences steady nuclear burning (Shen \& Bildsten 2007). Assuming an efficiency of $99 \%$, the mass-loss rate from the system is $3.1 \times 10^{-9} M_{\odot} \mathrm{yr}^{-1} \lesssim \dot{M} \lesssim 6.7 \times 10^{-9} M_{\odot} \mathrm{yr}^{-1} . \quad$ Typical speeds of the gas in the CSM are $100 \mathrm{~km} \mathrm{~s}^{-1} \lesssim v_{w} \lesssim 3000$ $\mathrm{km} \mathrm{s}^{-1}$. The lower part of the range is for steady nuclear burning. The highest speeds are relevant for systems with the highest accretion rates. Of particular interest is the speed for those systems with the lowest mass-loss rates, and they lose mass through the outer Lagrangian points at speeds up to $\sim 600 \mathrm{~km} \mathrm{~s}^{-1}$. We have marked this region in purple ("Outer Lagrangian losses") in Figure 5. With $\epsilon_{\mathrm{B}}=\epsilon_{\mathrm{e}}=0.1$, systems of this sort are ruled out for SN 2011fe and SN 2014J, and partly for SN $2012 \mathrm{cg}$, but not for the other SNe.

If the accretion rate is higher, i.e., $\dot{M}_{\text {acc }} \sim 6 \times 10^{-7} M_{\odot} \mathrm{yr}^{-1}$, winds around the WD are likely optically thick, limiting the accretion. Any further potential mass transfer will be lost from the system at an expected wind speed of order $10^{3} \mathrm{~km} \mathrm{~s}^{-1}$ (Hachisu et al. 1999, 2008). This is marked by the cyan-colored box in Figure 5. Assuming $\epsilon_{\mathrm{B}}=\epsilon_{\mathrm{rel}}=0.1, \mathrm{SN} 2011 \mathrm{fe}, \mathrm{SN} 2012 \mathrm{cg}$, and SN 2014J do not stem from such a type of progenitor system, while other $\mathrm{SNe}$ marked in the figure could.

Systems giving rise to recurrent novae are other possible SN Ia progenitors. These systems have a low accretion rate, $\dot{M}_{\text {acc }} \approx(1-3) \times 10^{-7} M_{\odot} \mathrm{yr}^{-1}$. At nova outbursts, they eject shells at speeds of a few $\times 1000 \mathrm{~km} \mathrm{~s}^{-1}$, with a time between shell ejections of a few or several years. From Table 3, the radio observations probe observing times between 2 and 20 days. For a model with N100, $s=2, \quad$ and $\dot{M}=1.0 \times 10^{-8} M_{\odot} \mathrm{yr}^{-1}\left(v_{w} / 100 \mathrm{~km} \mathrm{~s}^{-1}\right)$, the shock in our models reaches $\simeq 1.2 \times 10^{16} \mathrm{~cm}$. This constrains the presence of shells with recurrence times of $\lesssim 1.9\left(v_{\text {shell }} / 2000 \mathrm{~km} \mathrm{~s}^{-1}\right)^{-1}$ yr. Since the nova ejection is a transient event, the nova shell will be rather confined, and the likelihood for an SN shock being caught while interacting with a nova shell for the first $\sim 20$ days is small (about 30\%, according to Chomiuk et al. 2012). To estimate $\dot{M}$ during such a phase, we make use of the fact that models of recurrent novae predict that $\lesssim 15 \%$ of the accreted material between nova bursts is ejected (Yaron et al. 2005; Shen \& Bildsten 2009). We follow Chomiuk et al. (2012) and highlight the estimated range for $\dot{M}$ and $v_{w}$ with the 
Table 3

SNe Ia with the Most Constraining Data for a Wind-like Circumstellar Scenario

\begin{tabular}{|c|c|c|c|c|c|c|c|c|}
\hline SN Name & $\begin{array}{l}\text { Host and } \\
\text { Host Type }\end{array}$ & $\begin{array}{l}\text { Distance } \\
\text { (Mpc) }\end{array}$ & $\begin{array}{l}\text { Central } \\
\text { Freq. } \\
(\mathrm{GHz})\end{array}$ & $\begin{array}{c}\text { Time since } \\
\text { Explosion } \\
\text { (Days) }\end{array}$ & $\begin{array}{c}\text { Flux } \\
\text { Density }(1 \sigma) \\
(\mu \mathrm{Jy})\end{array}$ & $\begin{array}{c}\text { Luminosity } \\
\text { Upper Limit }(3 \sigma) \\
\left(10^{25} \mathrm{erg} \mathrm{s}^{-1} \mathrm{~Hz}^{-1}\right)\end{array}$ & $\begin{array}{c}\dot{M} / v_{w} \\
\text { Upper Limit } \\
\left(\frac{10^{-8} M_{\odot} \mathrm{yr}^{-1}}{100 \mathrm{~km} \mathrm{~s}^{-1}}\right)\end{array}$ & Reference \\
\hline SN 1995al & NGC 3021, SA(rs)bc & 27 & 1.4 & 17 & 80 & 6.98 & 4.0 & (1), (2) \\
\hline SN 2006X & NGC 4321. SAB(s)bc & 17 & 8.4 & 5.9 & 18 & 1.87 & 1.8 & (1) \\
\hline SN 2010fz & NGC 2967, SA(s)c & 31 & 6.0 & 8.9 & 9 & 3.11 & 3.0 & (1) \\
\hline SN $2011 \mathrm{dm}$ & UGC 11861, SABdm & 20 & 5.9 & 14.1 & 5 & 0.72 & 1.7 & (1) \\
\hline SN 2011ek & NGC 918, SAB(rs)c & 18 & 5.9 & 7.4 & 5 & 0.58 & 0.81 & (1) \\
\hline SN $2011 \mathrm{fe}$ & M101, SAB(rs)cd & 6.4 & 5.9 & 2.1 & 5.8 & 0.118 & 0.087 & (1), (3) \\
\hline SN 2011iv & NGC 1404, E1 & 19 & 6.8 & 6.2 & 18 & 3.11 & 2.3 & (1) \\
\hline SN $2012 Z$ & NGC 1309, SA(s)bc & 29 & 5.9 & 7.0 & 7 & 1.81 & 1.6 & (1) \\
\hline SN 2012cg & NGC 4424, SB(s)a & 15 & 4.1 & 5 & 5 & 0.40 & 0.35 & (1) \\
\hline SN 2016coj & NGC 4125, E pec & 20.1 & 1.51 & 11 & 42 & 6.09 & 2.8 & (4), (5) \\
\hline SN 2018gv & NGC $2525, \mathrm{SB}(\mathrm{s}) \mathrm{c}$ & 16.8 & 9.0 & 6 & 10 & 1.01 & 1.3 & (4), (6) \\
\hline SN 2018pv & NGC 3941, SB(s) & 13.1 & 5.1 & 14 & 19 & 1.18 & 2.1 & (4), (7) \\
\hline SN 2019np & NGC 3254, SA(s)bc & 22 & 1.51 & 10 & 22 & 3.82 & 1.7 & (4), (8) \\
\hline
\end{tabular}

Note. The columns, from left to right, are as follows: SN name; host galaxy and galaxy type; distance in Mpc; central frequency in GHz; mean observing epoch (in days since explosion); $1 \sigma$ flux density upper limits, in $\mu \mathrm{Jy}$; the corresponding $3 \sigma$ spectral luminosity in units of $10^{25} \mathrm{erg} \mathrm{s}^{-1} \mathrm{~Hz}^{-1}$; inferred $3 \sigma$ upper limit to the massloss rate in units of $10^{-8} M_{\odot} \mathrm{yr}^{-1}$, for an assumed wind velocity of $100 \mathrm{~km} \mathrm{~s}^{-1}$. (The values for $\dot{M}$ are for $\epsilon_{\mathrm{B}}=\epsilon_{\mathrm{e}}=0.1$.) References: (1) Chomiuk et al. 2016; (2) Panagia et al. 2006; (3) Pérez-Torres et al. 2014; (4) this paper; (5) Pérez-Torres et al. 2017; (6) Ryder et al. 2018; (7) Pérez-Torres et al. 2018; (8) Pérez-Torres et al. 2019.

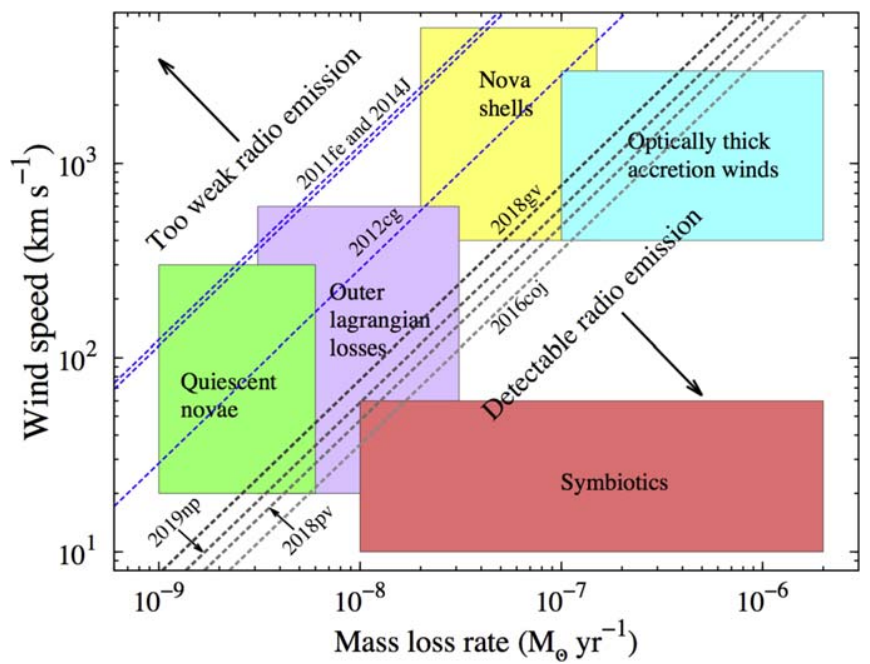

Figure 5. Constraints on the parameter space (wind speed vs. mass-loss rate) for SD scenarios for seven SNe Ia. The progenitor scenarios discussed in Section 5.2 are plotted as schematic zones, following Chomiuk et al. (2012) and Pérez-Torres et al. (2014). The $3 \sigma$ limits on $\dot{M} / v_{w}$ from Table 3 are marked by dashed lines, assuming $\epsilon_{\mathrm{B}}=\epsilon_{\mathrm{e}}=0.1, n=13, T_{\text {bright }}=5 \times 10^{10} \mathrm{~K}$, and the N100 explosion model. For the parameters in the upper left corner, i.e., low $\dot{M}$ and high $v_{w}$, the radio emission in the $s=2$ scenario is too weak to be detected, even for events like SN $2011 \mathrm{fe}$ and SN 2014J. The opposite is true for the lower right part of the figure. In particular, the $3 \sigma$ limits disfavor symbiotics as a likely progenitor channel. Not included in the figure is the spun-up/spun-down progenitor scenario (Di Stefano et al. 2011; Justham 2011), as this predicts a constant density. For SN $2011 \mathrm{fe}$ and SN $2014 \mathrm{~J}$, only a small part of the parameter space for likely SD progenitors is possible. yellow box in Figure 5. Using $\epsilon_{\mathrm{B}}=\epsilon_{\mathrm{e}}=0.1$, we cannot rule out nova shells completely for SN 2011fe and SN 2014J, and not at all for the other SNe.

The final box in Figure 5 is marked in green and is for novae during the quiescent phase between nova shell ejections. This is most likely for novae with long recurrence periods, and thus for those with the lowest accretion rates (i.e., $\left.\dot{M}_{\text {acc }} \sim 1 \times 10^{-7} M_{\odot} \mathrm{yr}^{-1}\right)$. The mass loss from the system is in this case $\dot{M} \sim 1 \times 10^{-9}\left(\epsilon_{\text {loss }} / 0.01\right)\left(v_{w} / 100 \mathrm{~km} \mathrm{~s}^{-1}\right)$. If $\epsilon_{\mathrm{B}}=\epsilon_{\mathrm{e}}=0.1$, the models rule out almost completely the scenario with WD accretion during the quiescent phase of the star for SN 2011fe and SN 2014J, whereas systems with the highest winds and lowest mass-loss rates are viable possibilities for the other SNe in Table 3.

For Figure 5 in general, the parameters in the upper left corner, i.e., low $\dot{M}$ and high $v_{w}$, the radio emission is too weak to be detected for any hitherto-observed SN Ia. The opposite is true for the lower right part of the figure, for which all the SNe Ia in Table 3 would have been detected if $\epsilon_{\mathrm{B}}=\epsilon_{\mathrm{e}}=0.1$, and if they would have belonged to any of the highlighted progenitor scenarios in Figure 5. In particular, for SN $2011 \mathrm{fe}$ and SN 2014J, only a small part of parameter space for possible SD progenitors is allowed.

\subsection{Microphysics Parameters $\epsilon_{B}$ and $\epsilon_{\text {rel }}$}

Progenitor constraints on the $\mathrm{SNe}$ in Table 3 were discussed in Section 5.2 under the assumption of $\epsilon_{\mathrm{B}}=\epsilon_{\mathrm{e}}=0.1$. This assumption has been used in most previous studies (e.g., Chomiuk et al. 2012, 2016; Pérez-Torres et al. 2014; Kundu et al. 2017), although cases with $\epsilon_{\mathrm{B}}=0.01$ have also been 
considered. A more general assumption is that $\epsilon_{\mathrm{B}}$ and $\epsilon_{\mathrm{e}}$ (and thus $\left.\epsilon_{\text {rel }}\right)$ can take any reasonable value, and this may differ from $\epsilon_{\mathrm{e}}=0.1$, in combination with $0.01 \leqslant \epsilon_{\mathrm{B}} \leqslant 0.1$.

As no SN Ia has yet been detected in the radio, observational constraints on $\epsilon_{\mathrm{B}}$ and $\epsilon_{\text {rel }}$ can only be obtained from corecollapse $\mathrm{SNe}$, preferably from stripped-envelope $\mathrm{SNe}$, as they have compact progenitors and fast SN ejecta. Assuming that all nonrelativistic electrons go into a power-law distribution with $\gamma_{\min } \geqslant 1$, Chevalier \& Fransson (2006) argued for $\epsilon_{\text {rel }} \geqslant 0.16\left(v_{s} / 5 \times 10^{4} \mathrm{~km} \mathrm{~s}^{-1}\right)^{-2}$ and used $\epsilon_{\mathrm{B}} \sim 0.1$. The question is whether the assumptions going into this are general. An example is the early-phase radio and X-ray emission of SN 2011dh. Soderberg et al. (2012; see also Krauss et al. 2012; Horesh et al. 2013) modeled this emission under the assumption of $0.1 \lesssim \epsilon_{\text {rel }} \lesssim 0.3$ and $0.01 \lesssim \epsilon_{\mathrm{B}} \lesssim 0.1$ and arrived at a wind density characterized by $\dot{M} \approx 6 \times 10^{-5} M_{\odot}$ $\mathrm{yr}^{-1}$ (for an assumed wind velocity of $v_{w}=1000 \mathrm{~km} \mathrm{~s}^{-1}$ ). This is a considerably less dense environment than estimated using models for the thermal X-ray emission from the $\mathrm{SN}$, at the somewhat later epoch of $\sim 500$ days, for which Maeda et al. (2014) and Kundu et al. (2019) estimate $\dot{M} \sim(2-4) \times 10^{-6} M_{\odot} \mathrm{yr}^{-1}$ (for $v_{w}=10 \mathrm{~km} \mathrm{~s}^{-1}$ ), i.e., $\sim 5$ times denser than that in the analysis of Soderberg et al. (2012). This could signal a decreased wind density toward the end of the life of the progenitor, but it may also be explained by too high values used by Soderberg et al. (2012) for $\epsilon_{\mathrm{B}}$ and $\epsilon_{\mathrm{rel}}$. Kundu et al. (2019) found good solutions to radio light curves at late epochs of the SNe IIb SN 1993J and SN 2011dh using $\epsilon_{\mathrm{B}}=\epsilon_{\mathrm{e}}=0.03$ and $\epsilon_{\mathrm{B}}=0.03$ and $\epsilon_{\mathrm{e}}=0.04$, respectively.

One can also gain information about microphysics parameters from the youngest SN Ia remnant detected in radio and X-rays, namely, G1.9+0.3 in the Milky Way (Condon et al. 1998; Reynolds et al. 2008). Models for its radio emission, assuming a constant-density medium around it, suggest the use of $\epsilon_{\text {rel }}=10^{-4}$ and $\epsilon_{\mathrm{B}} \sim 0.01$ (Sarbadhicary et al. 2019; see also below).

In Figure 6, we show solutions for several of the $\mathrm{SNe}$ in Table 3. The figure shows solutions for $\epsilon_{\mathrm{B}}$ and $\epsilon_{\text {rel }}$ for an assumed wind described by $\dot{M}=1.7 \times 10^{-8} M_{\odot} \mathrm{yr}^{-1}\left(v_{w} / 100 \mathrm{~km} \mathrm{~s}^{-1}\right)$, which corresponds to the upper left corner of the "Symbiotics" box in Figure 5. For combinations of $\epsilon_{\mathrm{B}}$ and $\epsilon_{\text {rel }}$ lying below and to the left of the solution curves, a symbiotic progenitor system cannot be excluded, based on the radio data in Table 3 alone. For our standard set of model parameters (i.e., $n=13, s=2$, $T_{\text {bright }}=5 \times 10^{10} \mathrm{~K}$, and the $\mathrm{N} 100$ explosion model) $\epsilon_{\mathrm{rel}}=\epsilon_{\mathrm{e}}$ in Figure 6 when $\epsilon_{\mathrm{B}} \gtrsim 0.1$, whereas for lower values of $\epsilon_{\mathrm{B}}$, $\epsilon_{\text {rel }}<\epsilon_{\mathrm{e}}$ (see Section 3). The vertical axis of the figure can also be used for $\epsilon_{\mathrm{e}}$ if one makes extrapolations toward smaller values of $\epsilon_{\mathrm{B}}$, like those extrapolations shown by dashed black lines for SN 2011fe and SN 2014J.

The horizontal blue dashed line highlights $0.01 \leqslant \epsilon_{\mathrm{B}} \leqslant 0.1$ for $\epsilon_{\text {rel }}=0.1$ In most analyses, only this small stretch in the $\epsilon_{\mathrm{B}}-\epsilon_{\text {rel }}$ plane is explored (e.g., Chomiuk et al. 2012, 2016; Pérez-Torres et al. 2014; Kundu et al. 2017), and, except in a few cases (e.g., Kundu et al. 2017), $\epsilon_{\text {rel }}$ is allowed to deviate from $\epsilon_{\mathrm{e}}$. Only solutions for SN 2011fe, SN 2012cg, and SN 2014J lie (in the case of SN 2012cg, marginally) below this blue region, which would mean that they cannot stem from symbiotic systems if $\epsilon_{\mathrm{rel}}=0.1$ and $\epsilon_{\mathrm{B}}>0.01$. However, for $\epsilon_{\mathrm{B}}=\epsilon_{\text {rel }}$ (i.e., relativistic particles and magnetic field strength being in equipartition), and both being $\lesssim 0.01$, symbiotic

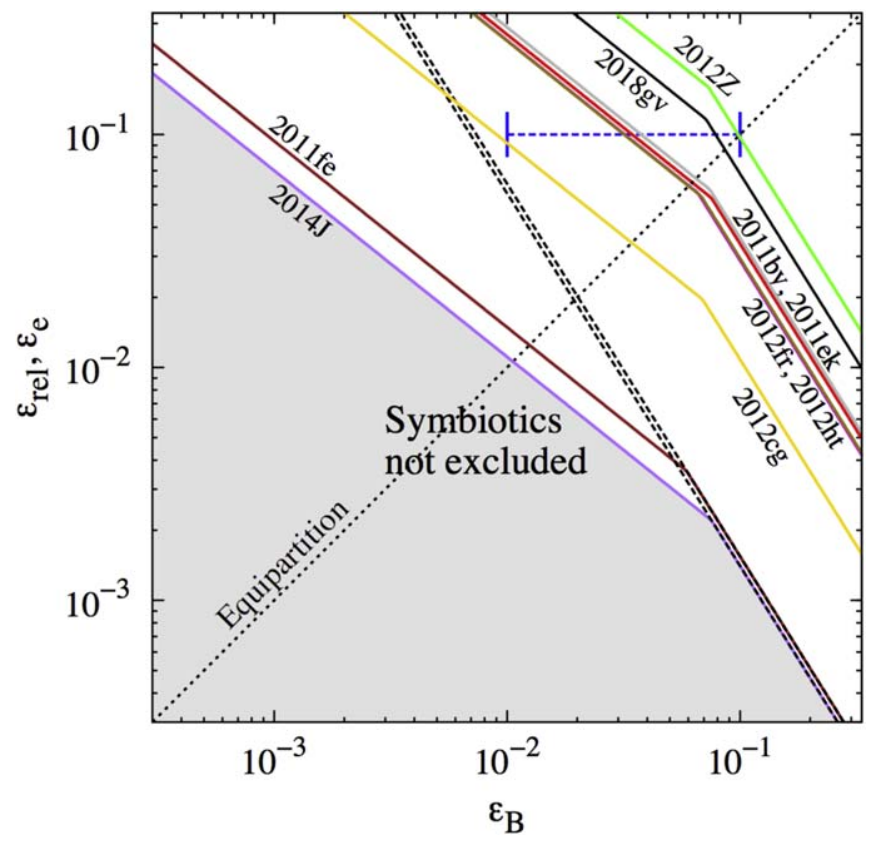

Figure 6. Parameter $\epsilon_{\mathrm{B}}$ vs. parameters $\epsilon_{\mathrm{e}}$ and $\epsilon_{\text {rel }}$ for the nine SNe Ia with the lowest estimated $\dot{M} / v_{w}$ in Table 3. The curve for each $\mathrm{SN}$ shows the combination of $\epsilon_{\mathrm{B}}$ and $\epsilon_{\mathrm{rel}}$ that gives an estimated $\dot{M} / \nu_{w}$ corresponding to the upper left corner in the "Symbiotics" box in Figure 5. For combinations of $\epsilon_{\mathrm{B}}$ and $\epsilon_{\text {rel }}$ below and to the left of these curves, symbiotic progenitor systems cannot be excluded. The gray area shows this parameter space for SN 2014J. The dashed lines for SN $2011 \mathrm{fe}$ and SN 2014J show $\epsilon_{\mathrm{B}}$ vs. $\epsilon_{\mathrm{e}}$ corresponding to the $\epsilon_{\mathrm{B}}$ vs. $\epsilon_{\mathrm{rel}}$ solutions for these $\mathrm{SNe}$. SNe toward the upper right corner are progressively less constraining with regard to symbiotics as a viable progenitor scenario. The blue dashed line depicts the range for $\epsilon_{\mathrm{B}}$ and $\epsilon_{\text {rel }}$ normally used in models for radio emission from SNe Ia, i.e., 0.01-0.1 for $\epsilon_{\mathrm{B}}$ and $\epsilon_{\text {rel }}=0.1$ (e.g., Chomiuk et al. 2012, 2016; Pérez-Torres et al. 2014). As can be seen, for this interval, symbiotics can be excluded only for SN 2011fe, SN 2012cg, and SN 2014J. See text for further details.

systems cannot be fully excluded, even for SN 2011fe and 2014J.

In Figure 7 we show a similar diagram for $n=13, s=0$, $T_{\text {bright }}=5 \times 10^{10} \mathrm{~K}$, and the merger explosion model. For SN 2011fe and SN 2014J we have used the $3 \mathrm{GHz} 3 \sigma$ upper limit at 1468 days and the $1.66 \mathrm{GHz} 3 \sigma$ upper limit at 410 days, respectively (Kundu et al. 2017). For G1.9+0.3, the $1.4 \mathrm{GHz}$ luminosity, at the estimated age of $125 \mathrm{yr}$, was used. This is $(6.4 \pm 0.3) \times 10^{22} \mathrm{ergs} \mathrm{s}^{-1} \mathrm{~Hz}^{-1}$ (Sarbadhicary et al. 2019). An interesting note is that a remnant elsewhere with such a luminosity could be detected with present-day instrumentation at distances $\lesssim 2.3 \mathrm{Mpc}$, assuming a $3 \sigma$ upper limit of $10 \mu \mathrm{Jy}$.

For our $s=0$ models of SN 2011fe, SN 2014J, and G1.9 +0.3 we have assumed densities of the circumstellar/ interstellar medium to be $0.1-1.0 \mathrm{~cm}^{-3}$, and we show the influence on the derived solutions for $\epsilon_{\mathrm{B}}$ and $\epsilon_{\mathrm{rel}}$ for this range in $n_{0}$ in Figure 7 for SN 2011fe. From this it can be seen that a density as low as $0.1 \mathrm{~cm}^{-3}$ would require high efficiency of magnetic field amplification and creation of relativistic particle energy density, so that both $\epsilon_{\mathrm{B}}$ and $\epsilon_{\mathrm{rel}}$ would have to be in excess of 0.1 (if in equipartition) to correspond to the observed radio upper limit. However, as discussed in Kundu et al. (2017), both SN 2011fe and SN 2014J are likely to have exploded in an interstellar region with a density of $\sim 1 \mathrm{~cm}^{-3}$. The solution for SN 2014J in Figure 7 is for that density. If any of those $\mathrm{SNe}$ would stem from a DD scenario, they would 


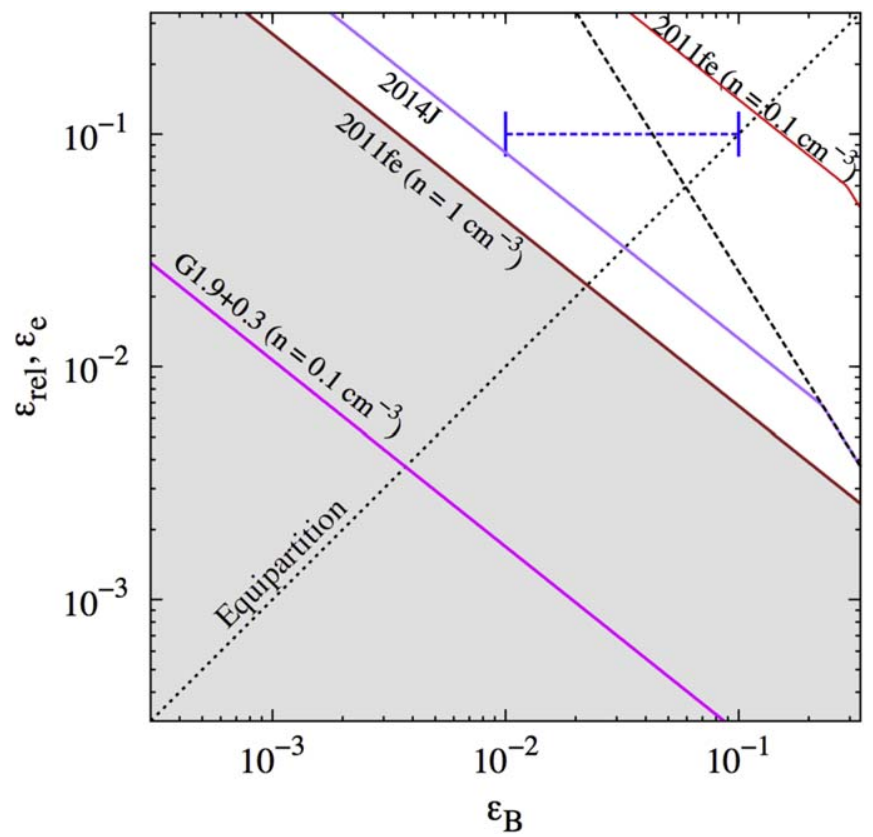

Figure 7. Parameter $\epsilon_{\mathrm{B}}$ vs. parameters $\epsilon_{\mathrm{e}}$ and $\epsilon_{\text {rel }}$ for SN 2011fe and SN 2014J, as well as the young SNR G1.9+03. The curves for SN $2011 \mathrm{fe}$ and SN 2014J show the combination of $\epsilon_{\mathrm{B}}$ and $\epsilon_{\mathrm{rel}}$ corresponding to an upper limit of a constant-density surrounding medium. For SN 2014J this limit is $n_{0}=1 \mathrm{~cm}^{-3}$, while for SN 2011fe we show models for both this density and $n_{0}=0.1 \mathrm{~cm}^{-3}$. For the detected radio emission from G1.9+03, we have used $n_{0}=0.1 \mathrm{~cm}^{-3}$. The dashed black line for SN 2014J has the same meaning as in Figure 6. Data are from Kundu et al. (2017) and Sarbadhicary et al. (2019). For combinations of $\epsilon_{\mathrm{B}}$ and $\epsilon_{\mathrm{rel}}$ below "2011fe $\left(n=1 \mathrm{~cm}^{-3}\right)$," a constant-density medium with $n_{0} \geqslant 1 \mathrm{~cm}^{-3}$ surrounding SN $2011 \mathrm{fe}$ cannot be excluded from radio observations alone. The blue dashed line has the same meaning as in Figure 6. See text for further details.

therefore indicate that the values for $\epsilon_{\mathrm{B}}$ and $\epsilon_{\mathrm{rel}}$ could be smaller than the standard range marked by the blue region in Figure 7.

A caveat with the model run for SN 2011fe exploding into a $\sim 1 \mathrm{~cm}^{-3}$ environment is that our assumption of $n=13$ only holds for maximum ejecta velocities of $\gtrsim 2.5 \times 10^{9} \mathrm{~cm} \mathrm{~s}^{-1}$. At lower velocities, the ejecta slope gets flatter (Kundu et al. 2017). A careful check shows that the maximum ejecta velocity is $\approx 3.05 \times 10^{9} \mathrm{~cm} \mathrm{~s}^{-1}$ at 1468 days for $n=13$ and $n_{0}=1 \mathrm{~cm}^{-3}$, so the solution for SN 2011fe in Figure 7 is not outside model bounds. However, for $\mathrm{G} 1.9+0.3$, with $n_{0}=0.1 \mathrm{~cm}^{-3}$ (as in Figure 7), $v_{s} \sim 1.45 \times 10^{9} \mathrm{~cm} \mathrm{~s}^{-1}$ (and the maximum ejecta velocity is $\approx 1.65 \times 10^{9} \mathrm{~cm} \mathrm{~s}^{-1}$ ) at $125 \mathrm{yr}$, which is at the base of the steep outer ejecta (see Figure 1 of Kundu et al. 2017). The observed velocities of the expanding radio structures are actually $\lesssim 10^{9} \mathrm{~cm} \mathrm{~s}^{-1}$ (Sarbadhicary et al. 2019), which means that the reverse shock has advanced deeper into the ejecta than in our model. Moreover, $T_{\text {bright }}$ is unlikely to remain constant over such a long period. Our model for G1.9 +0.3 should therefore only serve as rough estimates for $\epsilon_{\mathrm{B}}$ and $\epsilon_{\text {rel }}$. With this in mind, for $\epsilon_{\mathrm{B}}=0.02$ in Figure 7 we obtain $\epsilon_{\text {rel }} \sim 0.003$. In their models, more tuned to the remnant stage, Sarbadhicary et al. (2019) use $\epsilon_{\mathrm{rel}}=10^{-4}$ and $p=2.2$ to obtain a best fit for $n_{0}=0.18 \mathrm{~cm}^{-3}$. Although there is some controversy regarding the density around G1.9+0.3, probably in the range $0.02-0.3 \mathrm{~cm}^{-3}$ (Sarbadhicary et al. 2019, and references therein), densities much less than $n_{0} \sim 0.1 \mathrm{~cm}^{-3}$ may confront the apparent slow propagation of radio structures. It therefore seems reasonable to assume that $\epsilon_{\mathrm{B}}$ and $\epsilon_{\mathrm{rel}}$ are low for $\mathrm{G} 1.9+0.3$, as they also appear to be for slightly older remnants (e.g., Marcowith et al. 2016; Sarbadhicary et al. 2019).

In summary, both $\epsilon_{\mathrm{B}}$ and $\epsilon_{\text {rel }}$ are at present probably too uncertain to exclude most SD scenarios in Figure 5. If we use SN 2011dh as an example to constrain microphysics parameters for SNe Ia, we note that Soderberg et al. (2012) argue for $\epsilon_{\mathrm{B}}=0.01$ and $\epsilon_{\mathrm{e}} / \epsilon_{\mathrm{B}} \approx 30$ for that SN. Kundu et al. (2019) estimate a factor of $\sim 6.7$ higher circumstellar density than Soderberg et al. (2012) and therefore have to invoke less efficient radio production. If we assume $\epsilon_{\mathrm{e}} / \epsilon_{\mathrm{B}} \approx 30$, as did Soderberg et al. (2012), Equation (5) and the study of Kundu et al. (2019) suggest $\epsilon_{\mathrm{e}} \approx 0.11$ and $\epsilon_{\mathrm{B}} \approx 0.0036$ for SN 2011dh, rather than $\epsilon_{\mathrm{e}}=0.30$ and $\epsilon_{\mathrm{B}}=0.01$ argued for by Soderberg et al. (2012). If we further compensate for $T_{\text {bright }}=5 \times 10^{10} \mathrm{~K}$ used in the analysis here and $T_{\text {bright }}=4 \times 10^{10} \mathrm{~K}$ used by Kundu et al. (2019) for SN $2011 \mathrm{dh}, \epsilon_{\mathrm{e}} \approx 0.1$ and $\epsilon_{\mathrm{B}} \approx 0.0033$ may provide possible parameter values for $\mathrm{SNe}$ Ia. If we use those numbers in a model based on $\mathrm{N} 100$ and $n=13$, the upper limits on mass loss for SN 2011fe (SN 2014J) are $\dot{M} \approx 9.8(9.1) \times 10^{-9} M_{\odot}$ $\mathrm{yr}^{-1} \quad\left(\right.$ for $v_{w}=100 \mathrm{~km} \mathrm{~s}^{-1}$ ), and for SN 2012cg it is $\dot{M} \approx 3.9 \times 10^{-8} M_{\odot} \mathrm{yr}^{-1}$. This means that such a combination of $\epsilon_{\text {rel }}$ and $\epsilon_{\mathrm{B}}$ would fully rule out symbiotics for SN $2011 \mathrm{fe}$ and SN 2014J, but not for any of the other SNe in Table 3.

The uncertainty in especially $\epsilon_{\mathrm{B}}$ is not surprising from a theoretical point of view. Our current understanding of shock formation suggests the creation of intense turbulence with $\epsilon_{\mathrm{B}} \sim 0.01$ immediately behind the shock (Marcowith et al. 2016), but how this high level of turbulence can be maintained throughout the post-shock region is a conundrum. It may in fact be that the generation of magnetic field energy density is mainly driven by large-scale instabilities in connection with the contact discontinuity. If so, $\epsilon_{\mathrm{B}}$ would depend less on the conditions at the blast wave than on, e.g., the structure of the SN ejecta being overrun by the reverse shock (Björnsson \& Keshavarzi 2017). Spatially resolved studies, and modeling thereof, of young SNe like SN 1993J and young SNRs are essential to constrain this alternative.

\subsection{Other Clues to the Origin of SNe Ia}

In addition to radio emission, there are other clues to the origin of SNe Ia. Many of them involve circumstellar matter. We now discuss this, with emphasis on the SNe in Table 3.

\subsubsection{Circumstellar Absorption-line Features}

Among the SNe in Table 3, SN 2006X shows the clearest indication of a circumstellar medium, as it displayed timevariable narrow $\mathrm{Na}$ I D absorption features along the line of sight to the $\mathrm{SN}$, at a distance of $10^{16}-10^{17} \mathrm{~cm}$ from the progenitor system (Patat et al. 2007). In our models with N100, $\dot{M}=1 \times 10^{-8} M_{\odot} \mathrm{yr}^{-1}$ (and $v_{w}=100 \mathrm{~km} \mathrm{~s}^{-1}$ ), and $n=13$, interaction with such a shell would start between 17 and 215 days after the explosion. Radio observations of the SN, unfortunately, had a gap between days 18 and 287 (Chomiuk et al. 2016), so any temporary radio increase could have been missed, especially if the shell had modest thickness (see Harris et al. 2016, who constructed models for radio emission in shelllike media).

The presumed shell around SN 2006X would signal an SD scenario, but it does not have to be the result of a shell ejection. It could also exist in the so-called spun-up/spun-down super- 
Chandrasekhar mass WD scenario (Di Stefano et al. 2011; Justham 2011; Hachisu et al. 2012). Here the donor star shrinks far inside its Roche lobe prior to the explosion, and dilute circumstellar gas, with density similar to interstellar gas, would be expected close to the WD. If Roche lobe overflow ceased some $10^{3} \mathrm{yr}$ ago, and the wind speed of the nonconservative mass loss was $100 \mathrm{~km} \mathrm{~s}^{-1}$, dense circumstellar gas could reside at a distance of $\sim 3 \times 10^{17} \mathrm{~cm}$ and may explain the presumed shell around SN 2006X. A shell at such a distance from the SN would not be reached by the blast wave until after almost $3 \mathrm{yr}$ (using N100, $n=13$, and $n_{0}=1 \mathrm{~cm}^{-3}$ inside the shell). This is much later than the last radio observation of SN 2006X, performed on day 290, indicating a circumstellar density of $n_{0} \lesssim 50 \mathrm{~cm}^{-3}$, assuming $\epsilon_{\mathrm{B}}=\epsilon_{\mathrm{rel}}=0.1$ (Chomiuk et al. 2016).

Pinning down a possible increase in circumstellar density at $10^{17}-10^{18} \mathrm{~cm}$ from the SN was one of the motivations for the late-epoch observations of SN $2011 \mathrm{fe}$ and SN 2014J presented by Kundu et al. (2017). While SN 2011fe showed no obvious evidence of circumstellar shells (Patat et al. 2013), SN 2014J indeed displayed variations in narrow absorption of $\mathrm{K}$ I $\lambda 7665$ (Graham et al. 2015). However, the absorbing gas is at $\sim 10^{19}$ $\mathrm{cm}$ and is of interstellar origin (Maeda et al. 2016). As described in Kundu et al. (2017), no radio emission at late epochs was detected for SN 2014J, limiting the estimated circumstellar density to $n_{0} \lesssim 0.4 \mathrm{~cm}^{-3}$, assuming $\epsilon_{\mathrm{B}}=\epsilon_{\text {rel }}=0.1$ (Kundu et al. 2017). For such a circumstellar density, it would take $\gtrsim 200 \mathrm{yr}$ for the SN ejecta, using the $\mathrm{N} 100$ model, to reach a shell at $10^{19} \mathrm{~cm}$, i.e., the SN would then be in the SNR stage.

\subsubsection{Circumstellar Emission and Interaction}

A small fraction of $\mathrm{SNe}$ Ia show intense circumstellar interaction (see Section 1) and Balmer line emission. Graham et al. (2019) estimate that probably significantly less than $6 \%$ (Graham et al. 2019) have circumstellar shells within $<3 \times$ $10^{17} \mathrm{~cm}$ from the exploding star giving rise to such emission. The mass of these shells can be large, perhaps several solar masses (e.g., Hamuy et al. 2003; Aldering et al. 2006). This has been interpreted as clear evidence of SD progenitor systems for at least this fraction of SNe Ia.

As described in Sections 4.1.1 and 5.4.1, very few SNe Ia have been observed at depth at late epochs to possibly detect radio emission resulting from circumstellar interaction. Among those, SN 2006X and now recently SN 2015cp (Harris et al. 2018) show evidence of circumstellar interaction from observations at other wavelengths. As discussed in Section 5.4.1, the timing of the radio observations of SN 2006X may have been unfortunate; the importance of continuous radio monitoring of $\mathrm{SNe}$ Ia with circumstellar interaction was discussed by Chugai et al. (2004) for SN 2001ic, as well as by Harris et al. (2018) for SN 2015cp. Harris et al. $(2016,2018)$ model how the distribution of the circumstellar gas affects the expected radio emission.

An immediate method to probe circumstellar gas is through $\mathrm{X}$-ray observations, and the only SN Ia detected in X-rays is SN 2012ca (Bochenek et al. 2017). This SN belongs to the class of SN Ia $\mathrm{H} \alpha$ emitters, and the mass of the circumstellar shell is at least $0.1 \pm 0.05 M_{\odot}$. The relative proximity ( $\sim 80 \mathrm{Mpc})$ of SN 2012ca compared to, e.g., SN 2002ic and SN 2005gj is consistent with SN 2012ca being detected in $\mathrm{X}$-rays and the other two not (see Hughes et al. 2007).

\subsubsection{X-Ray Observations of SN 2011fe, SN 2012cg, and SN 2014J}

Margutti et al. $(2012,2014)$ provided deep X-ray limits for SN 2011fe and SN 2014J. The X-ray emission is for early epochs supposed to be due to inverse Compton scattering of photospheric photons on relativistic electrons in the shocked circumstellar gas. The derived limits on wind density do not depend on $\epsilon_{\mathrm{B}}$ but have an $\epsilon_{\mathrm{rel}}^{-2}$ dependence. Margutti et al. (2012)

$\dot{M} \lesssim 2 \times 10^{-9}\left(v_{w} / 100 \mathrm{~km} \mathrm{~s}^{-1}\right)\left(\epsilon_{\mathrm{rel}} / 0.1\right)^{-2} M_{\odot} \mathrm{yr}^{-1} \quad$ for SN 2011fe, and Margutti et al. (2014) find $\dot{M} \lesssim 1.2 \times 10^{-9}\left(v_{w} / 100 \mathrm{~km} \mathrm{~s}^{-1}\right)\left(\epsilon_{\mathrm{rel}} / 0.1\right)^{-2} M_{\odot} \mathrm{yr}^{-1}$ for SN 2014J. We can combine this with Equation (5) and entries in Table 3 for SN 2011fe and SN 2014J to get $\epsilon_{\mathrm{B}} \lesssim 0.03(0.06)$ for SN 2011fe (SN 2014J) for the X-ray upper limit to be stricter than the radio limit, assuming $\epsilon_{\mathrm{e}}=\epsilon_{\text {rel }}=0.1$ (where the first equality holds early in the evolution, i.e., when the most constraining X-ray observations were performed for these $\mathrm{SNe}$ ). For larger values of $\epsilon_{\mathrm{B}}$, radio is more constraining than $\mathrm{X}$-rays.

Recently, X-ray observations have also been reported for SN 2012cg (Shappee et al. 2018), and the absence of X-ray emission is claimed to provide an upper limit on $\dot{M}$, which is $\dot{M} \lesssim 1 \times 10^{-6}\left(v_{w} / 100 \mathrm{~km} \mathrm{~s}^{-1}\right)\left(\epsilon_{\mathrm{rel}} / 0.1\right)^{-2} M_{\odot} \mathrm{yr}^{-1}$. In the model used by Shappee et al. (2018) $\epsilon_{\text {rel }}$ is forced to have the same value as $\epsilon_{\mathrm{e}}$. However, at such high values of $\dot{M}$, our simulations with N100, $n=13$, and $t=5$ days give $\left(\epsilon_{\text {rel }} / 0.1\right)^{2} \simeq 0.27 \quad$ for $\dot{M}=4 \times 10^{-6}\left(v_{w} /\left(100 \mathrm{~km} \mathrm{~s}^{-1}\right)\right) M_{\odot} \mathrm{yr}^{-1}$, which should be a more correct upper limit of $\dot{M} / v_{w}$ from the absence of detected X-ray emission from SN $2012 \mathrm{cg}$.

The estimated limit on X-ray luminosity from SN $2012 \mathrm{cg}$, $L_{3-10 \mathrm{keV}}<1.4 \times 10^{39} \mathrm{erg} \mathrm{s}^{-1}$, is too high to be in conflict with the expected thermal X-ray emission for $\dot{M}=4 \times 10^{-6}\left(v_{w} /\left(100 \mathrm{~km} \mathrm{~s}^{-1}\right)\right) M_{\odot} \mathrm{yr}^{-1}$ (Lundqvist et al. 2013), but such a high mass-loss rate would have repercussions for interpretations of the radio data. While FFA is below unity $\left(\tau_{\mathrm{ff}} \sim 0.08\right.$; see Section 4.1$)$ for the $4.1 \mathrm{GHz}$ observations at 5 days (see Table 3), SSA would make the radio flux not peak until after $\sim 55$ days at $4.1 \mathrm{GHz}$, if $\epsilon_{\mathrm{e}}=\epsilon_{\mathrm{rel}}=0.1$. Despite SSA, the luminosity at 5 days is much higher than listed in Table 3. In order not to violate the observed $4.1 \mathrm{GHz}$ flux, $\epsilon_{\mathrm{B}} \lesssim 3 \times 10^{-6}$, assuming $\epsilon_{\mathrm{e}}=0.1$ and other model parameters for our $n=13$ and $s=2$ simulations. A combination of $\epsilon_{\text {rel }}=0.027$ and $\epsilon_{\mathrm{B}} \lesssim 3 \times 10^{-6}$ is probably extreme, and it is therefore most likely safe to assume that the X-ray observations of SN 2012cg are much less constraining than the radio data for this $\mathrm{SN}$ in terms of $\dot{M} / v_{w}$.

\subsubsection{Dust Extinction of SN 1989B, SN 2006X, SN 2012cg, SN 2012cu, and SN 2014J}

Circumstellar matter may reveal its presence through dust signatures. Among the $\mathrm{SNe}$ in Table 3, SN 2012cg, SN $2012 \mathrm{cu}$, and SN 2014J were investigated by Amanullah et al. (2015) to look for extinction features that could be due to circumstellar matter. For SN 2012cu and SN 2014J, no color evolution of the extinction was found, while for SN $2012 \mathrm{cg}$ there is evidence of some evolution. This could argue for circumstellar dust in SN 2012cg. However, when complementing with high-resolution data of $\mathrm{NaI} \mathrm{D,} \mathrm{Amanullah} \mathrm{et} \mathrm{al.}$ (2015) argue that any such dust around SN 2012cg must be at a distance of $\gtrsim 10^{19} \mathrm{~cm}$, which does not necessarily relate it to the 
progenitor system. The density probed by the published latest radio data, i.e., at 216 days, gives $n_{0} \lesssim 10 \mathrm{~cm}^{-3}$, assuming $\epsilon_{\mathrm{B}}=\epsilon_{\mathrm{rel}}=0.1$ (Chomiuk et al. 2016). Using the N100 model with $n=13$ and $n_{0}=10 \mathrm{~cm}^{-3}$, the blast wave had only expanded out to $\simeq 8 \times 10^{16} \mathrm{~cm}$ at that epoch, i.e., far inside the minimum distance to the dust.

In a more recent dust study, Bulla et al. (2018) analyze 48 reddened $\mathrm{SNe}$ Ia in order to localize sources of dust extinction. SNe appearing in both that study and Table 3 are SN 1989B, SN 2006X, SN 2012cu, and SN 2014J. From the models of Bulla et al. (2018), the distance between SN and dust for SN 1989B and SN 2012cu is $\geq 4.3 \times 10^{19} \mathrm{~cm}$ and $\gtrsim 1.0 \times 10^{19} \mathrm{~cm}$, respectively. For SN $2006 X$ and SN 2014J, the dust is mainly located $\sim 5 \times 10^{19} \mathrm{~cm}$ and $\sim 1.4 \times 10^{20} \mathrm{~cm}$ from the $\mathrm{SN}$, respectively. Only one $\mathrm{SN}$ in the study of Bulla et al. (2018), namely, SN 2003hx, has dust close enough to the $\mathrm{SN}, \sim 4 \times 10^{16} \mathrm{~cm}$, to argue for it being circumstellar. However, this $\mathrm{SN}$ is close to the center of its host galaxy, and Bulla et al. (2018) conclude that neither this nor any of the other SNe Ia in their study should be considered to harbor circumstellar dust. The dust is likely interstellar in all their cases.

Comparing with SNe in Table 3, we note that SN 2012cu was observed only once in radio, while SN 1989B was monitored until 114 days after the explosion. Chomiuk et al. (2016) estimate $n_{0} \lesssim 40 \mathrm{~cm}^{-3}$ for that epoch, assuming $\epsilon_{\mathrm{B}}=\epsilon_{\mathrm{rel}}=0.1$.

\subsubsection{Interaction with a Binary Companion}

In the SD scenario, the donor will be overrun by the $\mathrm{SN}$ blast wave in $\sim 0.6\left(v_{s} / 5 \times 10^{4} \mathrm{~km} \mathrm{~s}^{-1}\right)^{-1}\left(R_{\text {sep }} / 10^{13} \mathrm{~cm}\right) \mathrm{hr}$, where $R_{\text {sep }}$ is the separation between the donor and the WD at the time of explosion. The donor will therefore quickly be hidden inside the SN ejecta. However, during this early phase, and shortly thereafter, the donor can give rise to observational signatures in $\mathrm{X}$-rays and optical/UV, strength depending on the viewing angle (Kasen 2010). Caught early enough, $\sim 10 \%$ of SD cases should give rise to detectable signatures. In general, early interaction may create a light curve that would deviate from a single power law. Such cases have indeed been identified, e.g., SN 2012fr (Contreras et al. 2018), SN 2013dy (Zheng et al. 2013), SN 2014J (Goobar et al. 2015; Siverd et al. 2015), MUSSES1604D (Jiang et al. 2017), iPTF16abc (Miller et al. 2018), SN 2017cbv (Hosseinzadeh et al. 2017), and ASASSN18bt (Shappee et al. 2019). However, searches for other markers of SD origin have proven negative.

Of particular interest here are SN 2012fr and SN 2014J, which both are among the SNe Ia with the most constraining radio limits on circumstellar matter and microphysics parameters in the SD scenario (see Table 3 and Figure 6). This could signal that the early light-curve behavior is caused by something other than the ejecta-companion interaction.

A hint to another origin is the finding by Stritzinger et al. (2018) that there are two well-defined classes of SNe Ia, one of which has a blue color for the first few days, and the other a red color. In addition, there is a correlation between the early blue color and photospheric temperature at maximum light. At maximum, an SD companion should be well hidden by the SN ejecta, and the SN light is powered by radioactive decay. It is not clear why this should correlate with early blue color resulting from the ejecta-companion interaction. Further statistics is needed to shed light on this.

\subsubsection{Nebular Emission}

Long after the initial phases discussed in Section 5.4.5, an SD scenario donor may potentially reveal itself, but not until the optical depth through the ejecta has dropped for the donor material to become visible. In the 1D models of Mattila et al. (2005) and Lundqvist et al. (2013), this was calculated to occur after a few hundred days. In particular, lines of hydrogen, or perhaps helium, calcium, or oxygen (Lundqvist et al. 2015), with an expected velocity width of $\sim(0.5-2) \times 10^{3} \mathrm{~km} \mathrm{~s}^{-1}$ (e.g., Liu et al. 2012, 2013; Pan et al. 2012; Boehner et al. 2017 ) would indicate an SD scenario. The estimated amount of ablated gas from the donor varies depending on donor size and type and separation between the donor and the WD, but typical values are $\sim 0.01-0.1 M_{\odot}$.

Several studies have been done in the nebular phase of $\mathrm{SNe}$ Ia to look for material from a putative nondegenerate companion, using the models of Mattila et al. (2005) and Lundqvist et al. (2013), and in many cases the estimated upper limit of hydrogen mass from the companion is $\lesssim 0.01 M_{\odot}$ (e.g., Leonard 2007; Shappee et al. 2013, 2018; Lundqvist et al. 2015; Maguire et al. 2016). For our sample in Table 3, SN 2011ek, SN 2011fe, SN 2011iv, SN 2012cg, SN 2012cu, SN 2012fr, SN 2012ht, and SN 2014J have all been studied in the nebular phase, and the mass of hydrogen-rich donor material is $\lesssim 0.01 M_{\odot}$, except for SN $2012 \mathrm{cu}$, for which the limit is higher.

The most recent models for the expected emission from donor material in the nebular phase (Boehner et al. 2017; Sand et al. 2018; Dimitriadis et al. 2019; Tucker et al. 2019) indicate that the mass limits on ablated gas could be even lower than those derived from the models of Mattila et al. (2005) and Lundqvist et al. (2013). However, in all studies, systematic errors in the mass estimates could have been underestimated (Lundqvist et al. 2015), as the underlying SN spectrum can have intrinsic spectral features (e.g., Black et al. 2019) that may mask emission from ablated donor material. Time sequences of nebular spectra are needed to remove this uncertainty, as well as confusion due to other excitation mechanisms than radioactivity. This is highlighted by the claimed detection of ablated material in ASASSN-18tb (SN 2018fhw; Kollmeier et al. 2019) at a single epoch of around 155-160 days past explosion. A sequence of spectra of this event shows a persistent $\mathrm{H} \alpha$ emission already $\lesssim 60$ days (Valley et al. 2019) after explosion, which is more the hallmark of circumstellar interaction.

Despite some remaining uncertainty, the mass limits on ablated donor material from the absence of the nebular emission lines discussed in Lundqvist et al. (2015) are in conflict with the hydrodynamic models of the WD-companion interaction and pose a serious challenge to SD scenarios. The only possible SD scenario surviving this observational test may in fact be that of a spun-up/spun-down super-Chandrasekhar mass donor (see Lundqvist et al. 2015, for a discussion on this). From a circumstellar point of view, this would suggest that a constant circumstellar density out to some radius, corresponding to when mass transfer from the donor ceased (see Section 5.4.1), may provide the most likely circumstellar structure in the SD scenario. A low-density medium is also expected in the DD scenario. It may therefore not be surprising that $\mathrm{SNe}$ Ia are still undetected in radio. 


\subsubsection{SN 2013dy, SN 2016coj, SN 2018gv, SN 2018pv, and SN 2019np}

There is no evidence of circumstellar material in any of SN 2013dy, SN 2016coj, SN 2018gv, SN 2018pv, and SN 2019np. The most well-studied of them is SN 2013dy (Zheng et al. 2013; Pan et al. 2015; Zhai et al. 2016). It was detected $\sim 2.4 \mathrm{hr}$ after first light and had an abundance of unburned material in its envelope. $B$-band maximum occurred after $\sim 17.7$ days, and our radio observation was made $\sim 8$ days later. High-resolution optical spectra were obtained by Pan et al. (2015), but no variability was found in the standard absorption lines $\mathrm{Ca} \mathrm{H}$ and K, Na D $\lambda \lambda 5890$, 5896, and K I $\lambda \lambda 7665,7699$. These authors also show nebular spectra until 333 days after maximum, but no mass limits on possible donor material were presented.

SN 2016coj is estimated to have been detected $\sim 4.9$ days after first light (Zheng et al. 2017). It is a spectroscopically normal SN, with a $B$-band maximum at $\sim 16$ days. Highresolution spectra were obtained (Zheng et al. 2017), but owing to its $\sim 20 \mathrm{Mpc}$ distance, the signal-to-noise ratio was too low to identify any interstellar or circumstellar lines. SN 2016coj is the SN in our sample with the largest number of radio observations. They are, however, not as deep as for SN 2018gv, SN 2018pv, and SN 2019np.

Observations of SN 2018gv and SN 2018pv at other wavelengths than radio are discussed by P. Chen et al. (2020, in preparation). For SN 2018pv we have included ASAS-SN data (see Shappee et al. 2014, for a description of ASAS-SN). $B$-band data for SN 2019np have been estimated from data made available by N. Elias-Rosa and S. Dong. In Table 2 we have entered 6 days since explosion for SN 2018gv. This is a conservative estimate. From the optical data we have consulted, it may be closer to 5 days. This would push $\dot{M} / v_{w}$ close to $1.1 \times 10^{-8}\left(v_{w} / 100 \mathrm{~km} \mathrm{~s}^{-1}\right) M_{\odot} \mathrm{yr}^{-1}$. Likewise, the 14 and 10 days entered for SN 2018pv and SN 2019np, respectively, are conservative upper limits on the time since explosion.

Regarding other tests for the SD scenario, SN 2018gv, with its small host confusion, should be an excellent target for nebular emission studies. This could test the suggestion by Yang (2019) that this SN was indeed a member of an SD progenitor system, based on early ( -13.6 days with respect to $B$-band maximum light) spectropolarimetric measurements. The SN showed only $\leqslant 0.2 \%$ continuum polarization, as well as moderate line polarization, $0.46 \% \pm 0.04 \%$, for the strong Si II $\lambda 6355$ and $0.88 \% \pm 0.04 \%$ for the high-velocity Ca II component. Yang (2019) claims that this is inconsistent with a DD scenario. This is not in conflict with our radio limits (see Figure 5), if the progenitor was part of a symbiotic system, and/or at least one of $\epsilon_{\mathrm{e}}$ and $\epsilon_{\mathrm{B}}$ had a value $<0.1$.

\subsection{Future Radio Observations}

The deepest radio limits on circumstellar gas are for SN 2011fe and SN 2014J. A leap in sensitivity will occur when the Square Kilometre Array (SKA) comes online. In the SKA1-mid phase, a $1 \sigma$ sensitivity of $\sim 1.0 \mu \mathrm{Jy} \mathrm{beam}^{-1}$ can be reached in a $1 \mathrm{hr}$ integration at $1.4 \mathrm{GHz}$. The same limit is also expected at higher frequencies (e.g., 8.5 and $15 \mathrm{GHz}$ ). In Figure 8 we show a plot similar to that in Figure 4, but tuned to detection limits more relevant for SKA.

Judging from Figure 8, such a limit at $1.4 \mathrm{GHz}$ will probe $\dot{M} / v_{w}$ down to $\sim 3.7 \times 10^{-10}\left(v_{w} / 100 \mathrm{~km} \mathrm{~s}^{-1}\right) M_{\odot} \mathrm{yr}^{-1}$ for an

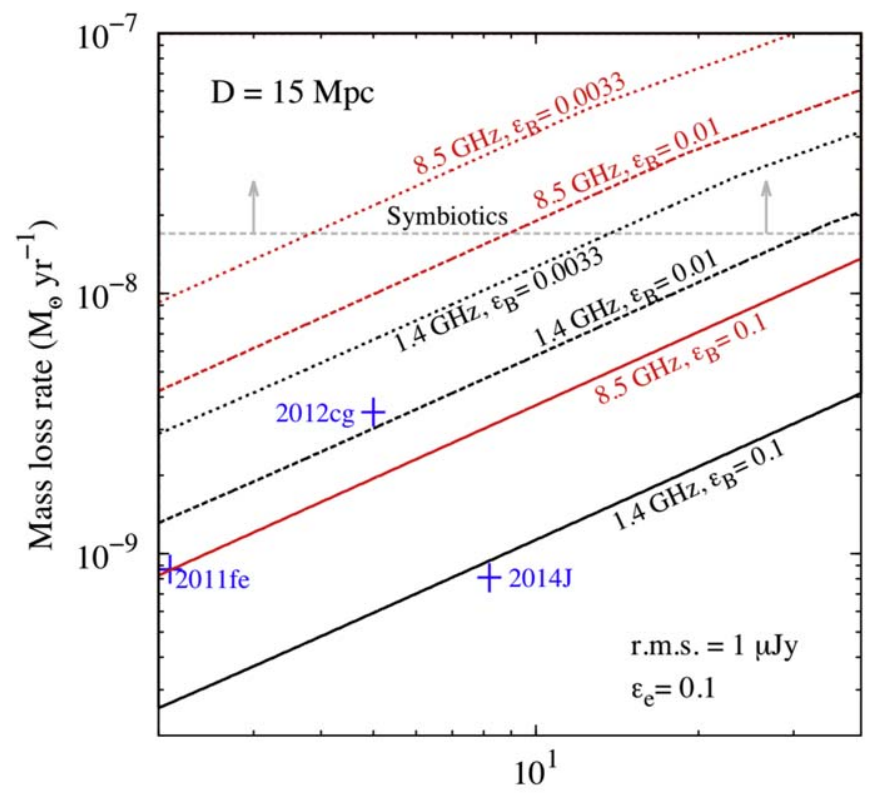

Time (days)

Figure 8. Same as Figure 4, but for the fixed rms of $1.0 \mu \mathrm{Jy}$ expected to be the sensitivity of SKA1-mid phase. Upper limits on mass-loss rate for two frequencies $(1.4$ and $8.5 \mathrm{GHz})$ are drawn for $3 \sigma$ flux limits. For each frequency solutions are made for three different values of $\epsilon_{\mathrm{B}}$, namely, 0.1 (solid lines), 0.01 (dashed lines), and 0.0033 (dotted lines). $\epsilon_{\mathrm{e}}=0.1$ for all models. Massloss rate limits for the hitherto most constraining events, SN 2011fe, SN $2012 \mathrm{cg}$, and SN 2014J, are marked in blue, assuming $\epsilon_{\mathrm{B}}=\epsilon_{\mathrm{e}}=0.1$. The expected lowest mass-loss rates for symbiotic systems are marked in gray (see Figure 5). See text for further details.

$\mathrm{SN}$ at $15 \mathrm{Mpc}$, observed 3 days after explosion, assuming the same model parameters used in Table 3. (The choice of 3 days after explosion in the estimate above rests on the currently planned cadence of the Large Synoptic Survey Telescope [LSST], which, like SKA, will be a southern hemisphere facility.) This is $\gtrsim 2.2-2.4$ times lower in $\dot{M} / v_{w}$ compared to the limits for SN 2011fe and SN 2014J in Table 3, despite those $\mathrm{SNe}$ Ia being much closer than $15 \mathrm{Mpc}$. For an $\mathrm{SN}$ at a distance of $20 \mathrm{Mpc}$, the limit on $\dot{M} / v_{w}$ would be $\sim 5.5 \times 10^{-10}\left(v_{w} / 100 \mathrm{~km} \mathrm{~s}^{-1}\right) M_{\odot} \mathrm{yr}^{-1}$. Judging from the VLA campaign by Chomiuk et al. (2016), mainly during 2011 and 2012 (see Table 3), we can expect approximately three to four SNe Ia per year within that distance, so a sample like that in Table 3 could be built in just a couple of years using SKA, and with limits on $\dot{M} / v_{w}$ that are almost a factor of two better than our current limits for SN 2011fe and SN 2014J, and $\sim 6$ times better than for SN 2012cg.

Alternatively, we will be able to constrain $\epsilon_{\mathrm{B}}$ and $\epsilon_{\text {rel }}$ to unprecedented levels. Figure 8 shows solutions not only for for $\epsilon_{\mathrm{B}}=0.1$ but also for $\epsilon_{\mathrm{B}}=0.0033$ and $\epsilon_{\mathrm{B}}=0.01$ (see Section 5.3). For an $\mathrm{SN}$ at $15 \mathrm{Mpc}$, observed at $1.4 \mathrm{GHz}$ 3 days after explosion, one would probe mass-loss rates down to $\dot{M} \sim 4.2 \times 10^{-9}\left(v_{w} / 100 \mathrm{~km} \mathrm{~s}^{-1}\right) M_{\odot} \mathrm{yr}^{-1}$, even if $\epsilon_{\mathrm{B}}=0.0033$. This would roughly correspond to the blue dashed line for SN 2012cg in Figure 5, but for $\epsilon_{\mathrm{B}}=0.0033$ instead of $\epsilon_{\mathrm{B}}=0.1$. For a distance of $5 \mathrm{Mpc}$ and $\epsilon_{\mathrm{B}}=0.0033$, one would probe down to $\dot{M} \sim 9.6 \times 10^{-10}\left(v_{w} / 100 \mathrm{~km} \mathrm{~s}^{-1}\right) M_{\odot} \mathrm{yr}^{-1}$, and one would essentially be bound to detect an SN Ia, if of SD origin. For SNRs, a remnant like G1.9+0.3 would be detected with SKA1mid at $3 \sigma$ out to $\sim 4 \mathrm{Mpc}$. 
A difference between Figures 4 and 8 is that $\dot{M}$ cannot be constrained in Figure 4 for short times since explosion and moderate flux limits, in particular for low frequencies. For deep flux limits like that in Figure 8 this is not a problem, as SSA is unimportant at the low mass-loss rates to be probed by SKA.

\section{Conclusions}

We report deep e-MERLIN and ATCA radio observations of the SNe Ia SN 2013dy, SN 2016coj, SN 2018gv, SN 2018pv, and SN 2019np, along with modeling of their radio emission. We do not detect the SNe. For the modeling we use the explosion model N100 (Röpke et al. 2012; Seitenzahl et al. 2013), in combination with a density $\rho \propto r^{-13}$ for the outermost $\mathrm{SN}$ ejecta. For the microphysical parameters $\epsilon_{\mathrm{e}}$ and $\epsilon_{\mathrm{B}}$ (which are the fractions of energy density of the shocked gas going into electrons with a power-law energy distribution and magnetic field strength, respectively) we first make the standard assumption $\epsilon_{\mathrm{e}}=\epsilon_{\mathrm{B}}=0.1$. Often it is assumed that $\epsilon_{\mathrm{e}}=\epsilon_{\text {rel }}$, where $\epsilon_{\mathrm{rel}}$ is the fraction of energy going into electrons with $\gamma_{\min } \gtrsim 1$. Following Kundu et al. (2017), we have relaxed that assumption in our models. With these considerations, we arrive at the upper limit on the mass-loss rate $\dot{M} \lesssim 12(2.8,1.3,2.1,1.7) \times 10^{-8}\left(v_{w} / 100 \mathrm{~km} \mathrm{~s}^{-1}\right) M_{\odot} \mathrm{yr}^{-1}$ in a wind scenario, for these five $\mathrm{SNe}$, respectively, where $v_{w}$ is the wind velocity of the mass loss from the progenitor system. The limits for SN 2016coj, SN 2018gv, SN 2018pv, and SN 2019 np are among the 16 deepest ever.

We have also compiled data for the $21 \mathrm{SNe}$ Ia with the lowest limits on $\dot{M} / v_{w}$ (including SN 2016coj, SN 2018gv, SN 2018pv, and SN 2019np), which from our models, with the same assumptions as above, all have $\dot{M} \lesssim 4 \times 10^{-8}\left(v_{w} / 100 \mathrm{~km} \mathrm{~s}^{-1}\right) M_{\odot} \mathrm{yr}^{-1}$. We compare those limits with the expected mass-loss rate in different SD progenitor scenarios. With $\epsilon_{\mathrm{e}}=\epsilon_{\mathrm{B}}=0.1$, the most nearby SNe in the sample, SN 2011fe and SN 2014J, are unlikely to be the result of SD progenitors, unless mass transfer from the donor ceased long before the explosion, like in the spun-up/ spun-down super-Chandrasekhar mass WD scenario. Alternatively, they are the results of two WDs merging, the so-called DD route. The latter is supported by the absence of detected $\mathrm{X}$-ray emission. As X-ray emission is expected to be due to inverse Compton scattering on relativistic electrons behind the SN blast wave, limits on $\dot{M} / v_{w}$ from X-rays depend on $\epsilon_{\text {rel }}$, but not on $\epsilon_{\mathrm{B}}$. Assuming that $\epsilon_{\mathrm{e}}=0.1$ and using $\dot{M} / v_{w}$ from X-ray limits, we obtain $\epsilon_{\mathrm{B}} \lesssim 0.03\left(3 \times 10^{-6}, 0.06\right)$ for SN 2011fe (SN 2012cg, SN 2014J), respectively, for the $\mathrm{X}$-ray upper limit to be stricter than the radio limit. The small value for SN 2012cg (which is the third most well-constrained $\mathrm{SN}$ Ia in radio) originates from a relatively poor X-ray limit on $\dot{M} / v_{w}$, which we have revised upward by a factor of four to $\dot{M} \lesssim 4 \times 10^{-6}\left(v_{w} / 100 \mathrm{~km} \mathrm{~s}^{-1}\right) M_{\odot} \mathrm{yr}^{-1}$.

We caution that the uncertainty in the microphysical parameters (mainly $\epsilon_{\mathrm{B}}$ ) makes limits on $\dot{M} / v_{w}$ from radio somewhat difficult to judge. To study this, we have allowed $\epsilon_{\text {rel }}$ and $\epsilon_{\mathrm{B}}$ to take any plausible values. In particular, we have tested what is the allowed range in $\epsilon_{\mathrm{rel}}$ and $\epsilon_{\mathrm{B}}$ for the $21 \mathrm{SNe} \mathrm{Ia}$ in our sample, for them not to stem from symbiotic progenitor systems, which we have defined to have a minimum mass-loss rate of $\dot{M} \gtrsim 1.7 \times 10^{-8}\left(v_{w} / 100 \mathrm{~km} \mathrm{~s}^{-1}\right) M_{\odot} \mathrm{yr}^{-1}$. Symbiotic systems are those of the likely progenitors with the largest $\dot{M} / v_{w}$. Assuming $\epsilon_{\text {rel }}=0.1$, and judging from radio alone, the progenitors of even SN 2011fe and SN 2014J could have had such high mass-loss rates for $\epsilon_{\mathrm{B}} \lesssim 10^{-3}$. However, $\dot{M} \gtrsim 1.7 \times 10^{-8}\left(v_{w} / 100 \mathrm{~km} \mathrm{~s}^{-1}\right) M_{\odot} \mathrm{yr}^{-1}$ is ruled out from $\mathrm{X}$-ray nondetections for these $\mathrm{SNe}$, if $\epsilon_{\text {rel }}=0.1$. A combination with relativistic electrons and the magnetic field strength in equipartition, so that $\epsilon_{\text {rel }}=\epsilon_{\mathrm{B}}=0.01$, could make symbiotic progenitors for SN 2011fe and SN 2014J with $\dot{M} \gtrsim 1.7 \times 10^{-8}\left(v_{w} / 100 \mathrm{~km} \mathrm{~s}^{-1}\right) M_{\odot} \mathrm{yr}^{-1}$ pass observational tests in radio and X-rays. For $\mathrm{SN} 2012 \mathrm{cg}, \epsilon_{\text {rel }}=0.1$ and $\epsilon_{\mathrm{B}}=0.01$ is enough to rule out a symbiotic progenitor, while for other $\mathrm{SNe}$ in the sample, radio limits cannot rule out symbiotic progenitor systems, let alone other SD channels with lower mass-loss rates, even if $\epsilon_{\mathrm{B}}$ is as high as $\sim 0.04$ (assuming $\left.\epsilon_{\text {rel }}=0.1\right)$.

To draw conclusions on progenitor origin from radio and $\mathrm{X}$-rays, it is thus imperative to know the microphysical parameters. Information can be provided by objects with actual detections. One is the youngest SN Ia remnant detected in radio, G1.9+0.3. Although there is some debate regarding the density around G1.9+0.3, its detection at an age of $\sim 125 \mathrm{yr}$ points toward $\epsilon_{\mathrm{rel}}$ and $\epsilon_{\mathrm{B}}$ both being of order 0.01 , or less. With such numbers for SN 2011fe and SN 2014J at late epochs (i.e., $t=1-3 \mathrm{yr}$ ), as well as the $21 \mathrm{SNe}$ Ia in our sample at early epochs, it comes as no surprise that there are yet no radio detections of SNe Ia, or young SN Ia remnants, besides local events like G1.9+0.3, and possibly SN 1885A (Sarbadhicary et al. 2019).

Estimates of $\epsilon_{\text {rel }}$ and $\epsilon_{\mathrm{B}}$ can also be obtained from strippedenvelope core-collapse SNe. We have highlighted SN 2011dh as an example and argue for $\epsilon_{\mathrm{e}} \approx 0.1$ and $\epsilon_{\mathrm{B}} \approx 0.0033$. Such a combination would fully rule out symbiotics for SN $2011 \mathrm{fe}$ and SN 2014J, but not for any of the other SNe Ia.

When radio observations of a newly detected SN Ia are being planned, it is crucial to take into account SSA. As we show in Figure 4 , too early low-frequency $(\lesssim 2 \mathrm{GHz})$ observations may lead to no constraints on circumstellar matter if the $3 \sigma$ flux limit is too high and/or the observations are being performed too early. SN 2019np serves as an example, where $1.28 \mathrm{GHz}$ observations at $t=7$ days $(3 \sigma$ limit of $57 \mu \mathrm{m})$ give no solution for $\dot{M} / v_{w}$, and $1.51 \mathrm{GHz}$ observations at $t=10$ days $(3 \sigma$ limit of $66 \mu \mathrm{m})$ can be used to rule out $1.7 \times 10^{-8} M_{\odot} \mathrm{yr}^{-1} \lesssim \dot{M}\left(v_{w} / 100 \mathrm{~km} \mathrm{~s}^{-1}\right)^{-1} \lesssim-$

$2.4 \times 10^{-7} M_{\odot} \mathrm{yr}^{-1}$ (using our standard parameters for the SN dynamics and $\left.\epsilon_{\mathrm{rel}}=\epsilon_{\mathrm{B}}=0.1\right)$. To rule out also $\gtrsim 2.4 \times 10^{-7} M_{\odot} \mathrm{yr}^{-1}, \quad$ complementary observations are needed. Considering SSA will be important when SKA becomes operational, and if it will be used to observe moderately distant ( $\gtrsim 40-50 \mathrm{Mpc}$ ) SNe Ia at low frequencies at early epochs. For more nearby SNe Ia, SSA is less important for SKA (see Figure 8), and one should in just a few years create a significantly better sample than discussed here.

While radio and X-rays are important probes for circumstellar matter, other tools are also needed to pin down the origin of SNe Ia, in particular observations in the optical and infrared. Current evidence points in favor of DD being responsible for the majority of normal SNe Ia, with the strongest evidence, besides no detected radio or X-ray emission, being no circumstellar dust in any SN Ia (Bulla et al. 2018), no trace of donor material in nebular spectra (e.g., Lundqvist et al. 2015; Maguire et al. 2016; Sand et al. 2018; Tucker et al. 2019), and tight constraints on donor size from the very early interaction between SN ejecta and a donor (e.g., Kasen 2010; Shappee et al. 2018). Evidence for circumstellar matter in 
normal SNe Ia is provided by time-varying absorption lines in a few SNe Ia (e.g., Patat et al. 2007), and emission lines in one case (Graham et al. 2019), and observing such SNe in the radio, at moderate cadence, may provide the best prospects of detecting radio emission from an SN Ia in the near future.

We thank Subo Dong for sharing data for SN 2018gv and SN 2018pv prior to publication, Stephen Reynolds for discussions and comments on an early version of the manuscript, and Nancy Elias-Rosa for discussions on SN 2019np. P. L. acknowledges support from the Swedish Research Council, and M.P.T. acknowledges financial support from the Spanish MCIU through the "Center of Excellence Severo Ochoa" award for the Instituto de Astrofísica de Andalucía (SEV-2017-0709) and through the MINECO grants AYA2012-38491-C02-02 and AYA2015-63939-C2-1-P. J.M. acknowledges financial support from the State Agency for Research of the Spanish MCIU through the "Center of Excellence Severo Ochoa" award to the Instituto de Astrofísica de Andalucía (SEV-2017-0709) and from the grant RTI2018-096228-B-C31 (MICIU/FEDER, EU). The research leading to these results has received funding from the European Commission Horizon 2020 Research and Innovation Programme under grant agreement No. 730562 (RadioNet).

The electronic Multi-Element Radio Linked Interferometer Network (e-MERLIN) is the UK's facility for high-resolution radio astronomy observations, operated by the University of Manchester for the Science and Technology Facilities Council (STFC). The Australia Telescope Compact Array (ATCA) is part of the Australia Telescope National Facility, which is funded by the Australian Government for operation as a National Facility managed by CSIRO. The ATCA data reported here were obtained under program C1303 (P.I. P. Lundqvist). This research has made use of the NASA Astrophysics Data System (ADS) Bibliographic Services and the NASA/IPAC Extragalactic Database (NED), which is operated by the Jet Propulsion Laboratory, California Institute of Technology, under contract with the National Aeronautics and Space Administration.

Facilities: e-MERLIN, ATCA, JVLA, AMI, MeerKAT.

Software: CASA (McMullin et al. 2007), AIPS (Wells 1985), MIRIAD (Sault et al. 1995), e-MERLIN pipeline (Argo 2015).

\section{ORCID iDs}

Peter Lundqvist (iD https://orcid.org/0000-0002-3664-8082 Esha Kundu (iD https://orcid.org/0000-0002-4807-379X Miguel A. Pérez-Torres (ib https://orcid.org/0000-00015654-0266

Stuart D. Ryder (iD https://orcid.org/0000-0003-4501-8100 Claes-Ingvar Björnsson (iD https://orcid.org/0000-00023284-4481

Javier Moldon (iD https://orcid.org/0000-0002-8079-7608

Megan K. Argo (iD https://orcid.org/0000-0003-3594-0214

Erik C. Kool (i) https://orcid.org/0000-0002-7252-3877

\section{References}

Aldering, G., Antilogus, P., Bailey, S., et al. 2006, ApJ, 650, 510 Amanullah, R., Johansson, J., \& Goobar, A. 2015, MNRAS, 453, 3300 Argo, M. K. 2015, JORS, 3, e2

Björnsson, C.-I., \& Keshavarzi, S. T. 2017, ApJ, 841, 12

Björnsson, C.-I., \& Lundqvist, P. 2014, ApJ, 787, 143

Black, C. S., Fesen, R. A., \& Parrent, J. T. 2019, MNRAS, 483, 1114
Bochenek, C. D., Dwarkadas, V. V., Silverman, J. M., et al. 2017, MNRAS, 473, 336

Boehner, P., Plewa, T., \& Langer, N. 2017, MNRAS, 465, 2060

Branch, D., Livio, M., Yungelson, L. R., Boffi, F. R., \& Baron, E. 1995, PASP, 107, 1019

Bufano, F., Berton, M., Vogl, C., et al. 2018, ATel, 11177, 1

Bufano, F., Pignata, G., Bersten, M., et al. 2014, MNRAS, 439, 1807

Bulla, M., Goobar, A., \& Dhawan, S. 2018, MNRAS, 479, 3663

Casper, C., Zheng, W., Li, W., et al. 2013, CBET, 3588, 1

Chevalier, R. A. 1982a, ApJ, 258, 790

Chevalier, R. A. 1982b, ApJ, 259, 302

Chevalier, R. A., \& Fransson, C. 2006, ApJ, 651, 381

Chomiuk, L., Soderberg, A. M., Chevalier, R. A., et al. 2016, ApJ, 821, 119

Chomiuk, L., Soderberg, A. M., Moe, M., et al. 2012, ApJ, 750, 164

Chugai, N. N., Chevalier, R. A., \& Lundqvist, P. 2004, MNRAS, 355, 627

Condon, J. J., Cotton, W. D., Greisen, E. W., et al. 1998, AJ, 115, 1693

Contreras, C., Phillips, M. M., Burns, C. R., et al. 2018, ApJ, 859, 24

Di Stefano, R., Voss, R., \& Clayes, J. S. W. 2011, ApJL, 738, L1

Dilday, B., Howell, D. A., Cenko, S. B., et al. 2012, Sci, 337, 942

Dimitriadis, G., Rojas-Bravo, C. D., Kilpatrick, R. J., et al. 2019, ApJL, 870, L14

Goobar, A., Kromer, M., Siverd, R., et al. 2015, ApJ, 799, 106

Graham, M. L., Harris, C. E., Nugent, P. E., et al. 2019, ApJ, 871, 1

Graham, M. L., Valenti, S., Fulton, B., et al. 2015, ApJ, 810, 136

Hachisu, I., Kato, M., \& Nomoto, K. 1999, ApJ, 522, 487

Hachisu, I., Kato, M., \& Nomoto, K. 2008, ApJ, 679, 1390

Hachisu, I., Kato, M., Saio, H., \& Nomoto, K. 2012, ApJ, 744, 69

Hamuy, M., Phillips, M. M., Suntzeff, N. B., et al. 2003, Natur, 424, 651

Hancock, P. J., Gaensler, B. M., \& Murphy, T. 2011, ApJ, 735, 35

Harris, C. E., Nugent, P. E., \& Horesh, J. D. 2018, ApJ, 868, 21

Harris, C. E., Nugent, P. E., \& Kasen, D. N. 2016, ApJ, 823, 100

Heywood, I., Horesh, A., van der Horst, A. J., et al. 2019, ATel, 12416, 1

Horesh, A., Kulkarni, S. R., Fox, D. B., et al. 2012, ApJ, 746, 21

Horesh, A., Stockdale, C., Fox, D. B., et al. 2013, MNRAS, 436, 1258

Hosseinzadeh, G., Sand, D. J., Valenti, S., et al. 2017, ApJL, 845, L11

Hoyle, F., \& Fowler, W. A. 1960, ApJ, 132, 565

Hughes, J. P., Chugai, N., Chevalier, R., Lundqvist, P., \& Schlegel, E. 2007, ApJ, 670, 1260

Iben, I., Jr., \& Tutukov, A. V. 1984, ApJS, 54, 335

Jiang, J.-A., Doi, M., Maeda, K., et al. 2017, Natur, 550, 80

Justham, S. 2011, ApJL, 730, L34

Kasen, D. 2010, ApJ, 708, 1025

Kollmeier, J. A., Chen, P., Dong, S., et al. 2019, MNRAS, 486, 3041

Krauss, M. I., Soderberg, A. M., Chomiuk, L., et al. 2012, ApJ, 750, 40

Kundu, E., Lundqvist, P., Pérez-Torres, M. A., Herrero-Illana, R., \& Alberdi, A. 2017, ApJ, 842, 17

Kundu, E., Lundqvist, P., Sorokina, E., et al. 2019, ApJ, 875, 17

Leonard, D. C. 2007, ApJ, 670, 1275

Liu, Z. W., Pakmor, R., Röpke, F. K., et al. 2012, A\&A, 548, A2

Liu, Z. W., Pakmor, R., Seitenzahl, I. R., et al. 2013, ApJ, 774, 37

Lundqvist, P., Mattila, S., Sollerman, J., et al. 2013, MNRAS, 435, 329

Lundqvist, P., Nyholm, A., Taddia, F., et al. 2015, A\&A, 577, A39

Maeda, K., Katsuda, S., Bamba, A., et al. 2014, ApJ, 785, 95

Maeda, K., Tajitsu, A., Kawabata, K., et al. 2016, ApJ, 816, 57

Maguire, K., Taubenberger, S., Sullivan, M., \& Mazzali, P. A. 2016, MNRAS, 457, 325

Maoz, D., Mannucci, F., \& Nelemans, G. 2014, ARA\&A, 52, 107

Marcowith, A., Bret, A., \& Bykov, A. 2016, RPPh, 79, 046901

Margutti, R., Parrent, J., Kamble, A., et al. 2014, ApJ, 790, 52

Margutti, R., Soderberg, A. M., Chomiuk, L., et al. 2012, ApJ, 751, 134

Mattila, S., Lundqvist, P., Sollerman, J., et al. 2005, A\&A, 443, 649

McMullin, J. P., Waters, B., Schiebel, D., Young, W., \& Golap, K. 2007, in ASP Conf. Ser. 376, Astronomical Data Analysis Software and Systems XVI, ed. R. A. Shaw, F. Hill, \& D. J. Bell (San Francisco, CA: ASP), 127 Miller, A. A., Cao, Y., Piro, A. L., et al. 2018, ApJ, 852, 100 Mooley, K. P., Fender, R. P., Cantwell, T., et al. 2016, ATel, 9193, 1 Nomoto, K. 1982, ApJ, 253, 798

Nomoto, K., Saio, H., Kato, M., \& Hachisu, I. 2007, ApJ, 663, 1269

Pakmor, R., Kromer, M., Taubenberger, S., et al. 2012, ApJL, 747, L10

Pan, K.-C., Ricker, P. M., \& Taam, R. E. 2012, ApJ, 750, 151

Pan, Y. C., Foley, R. J., Kromer, M., et al. 2015, MNRAS, 452, 4307 Panagia, N., Van Dyk, S. D., Weiler, K. W., et al. 2006, ApJ, 646, 369 Patat, N., Chandra, P., Chevalier, R., et al. 2007, Sci, 317, 924

Patat, N., Cordiner, M. A., Cox, N. L. J., et al. 2013, A\&A, 549, 62 Pérez-Torres, M., Argo, M., Lundqvist, P., et al. 2013, ATel, 5619, 1 Pérez-Torres, M., Lundqvist, P., Kundu, E., et al. 2017, ATel, 10168, 1 
Pérez-Torres, M., Lundqvist, P., Moldon, J., et al. 2018, ATel, 11324, 1 Pérez-Torres, M., Lundqvist, P., Moldon, J., et al. 2019, ATel, 12411, 1 Pérez-Torres, M. A., Lundqvist, P., \& Beswick, R. J. 2014, ApJ, 792, 38 Perlmutter, S., Aldering, G., Goldhaber, G., et al. 1999, ApJ, 517, 565

Rebassa-Mansergas, A., Toonen, S., Korol, V., \& Torres, S. 2019, MNRAS, 482, 3656

Reynolds, S. P., Borkowski, K. J., Green, D. A., et al. 2008, ApJL, 680, L41

Riess, A., Filippenko, A. V., Challis, P., et al. 1998, AJ, 116, 1009

Röpke, F. K., Kromer, M., Seitenzahl, I. R., et al. 2012, ApJL, 750, L19

Russell, B. R., \& Immler, S. 2012, ApJL, 748, L29

Ryder, S. D., Lundqvist, P., Pérez-Torres, M. A., et al. 2018, ATel, 11211, 1

Sand, D. J., Graham, M. L., Botyánszki, J., et al. 2018, ApJ, 863, 24

Sarbadhicary, S. K., Chomiuk, L., Badenes, C., et al. 2019, ApJ, 872, 191

Sault, R. J., Teuben, P. J., \& Wright, M. C. H. 1995, in ASP Conf. Ser. 77, Astronomical Data Analysis Software and Systems IV, ed. R. Shaw, H. E. Payne, \& J. J. E. Hayes (San Francisco, CA: ASP), 433

Seitenzahl, I. R., Ciaraldi-Schoolmann, F., Röpke, F. K., et al. 2013, MNRAS, 429, 1156

Shappee, B. J., Piro, A. L., \& Stanek, S. G. 2018, ApJ, 855, 6

Shappee, B. J., Prieto, J. L., Grupe, D., et al. 2014, ApJ, 788, 48

Shappee, B. J., Stanek, K. Z., Pogge, R. W., \& Garnavich, P. M. 2013, ApJL, 762, L5

Shappee, B. J., Holoien, T. W.-S., Drout, M. R., et al. 2019, ApJ, 870, 13

Shen, K. J., \& Bildsten, L. 2007, ApJ, 660, 1444
Shen, K. J., \& Bildsten, L. 2009, ApJ, 692, 324

Siebert, M. R., Dimitriadis, G., \& Foley, R. J. 2018, ATel, 11175, 1

Simon, J. D., Gal-Yam, A., Gnat, O., et al. 2009, ApJ, 702, 1157

Siverd, R. J., Goobar, A., Stassun, K. G., \& Pepper, J. 2015, ApJ, 799, 105

Soderberg, A. M., Margutti, R., Zauderer, B. A., et al. 2012, ApJ, 752, 78

Stritzinger, M. D., Shappee, B. J., Piro, A. L., et al. 2018, ApJL, 864, L35

Thompson, T. A. 2011, ApJ, 741, 82

Tucker, M. A., Shappee, B. J., \& Wisniewski, J. P. 2019, ApJL, 872, L22

Tully, R. B., Courtois, H. M., Dolphin, A. E., et al. 2013, AJ, 146, 86

Tutukov, A. V., \& Yungelson, L. R. 1979, AcA, 29, 665

Valley, P. J., Fausnaugh, M., Jha, S. W., et al. 2019, MNRAS, 487, 2372

Wang, B. 2018, RAA, 18, 49

Webbink, R. F. 1984, ApJ, 277, 355

Wells, D. C. 1985, in Ettore Majorana International Science Series, Data Analysis in Astronomy, ed. V. di Gesu (New York: Plenum Press), 195

Whelan, J., \& Iben, I., Jr. 1973, ApJ, 186, 1007

Yamanaka, M., Kawabata, M., Nakaoka, T., \& Huang, R. 2018, ATel, 11278,1

Yang, Y. 2019, AAS Meeting, 233, 258.08

Yaron, O., Prialnik, D., Shara, M. M., \& Kovetz, A. 2005, ApJ, 623, 398

Zhai, Q., Zhang, J.-J., Wang, X.-F., et al. 2016, AJ, 151, 125

Zheng, W., Filippenko, A. V., Mauerhan, J., et al. 2017, ApJ, 841, 64

Zheng, W., Silverman, J. M., Filippenko, A. V., et al. 2013, ApJL, 778, L15

Zheng, W., Yuk, H., Manzano-King, G., et al. 2016, ATel, 9095, 1 